\title{
Modified Desolvation Method Enables Simple One-Step Synthesis of Gelatin Nanoparticles from Different Gelatin Types with Any Bloom Values
}

\author{
Pavel Khramtsov ${ }^{1,2,3, *}$, , Oksana Burdina ${ }^{2}$, Sergey Lazarev ${ }^{2}\left(\mathbb{0}\right.$, Anastasia Novokshonova ${ }^{2}$, Maria Bochkova ${ }^{1,2}$, \\ Valeria Timganova ${ }^{1}{ }^{(}$, Dmitriy Kiselkov ${ }^{4}$, Artem Minin ${ }^{5,6} \oplus^{\circ}$, Svetlana Zamorina ${ }^{1,2}$ and Mikhail Rayev ${ }^{1,2}$ \\ 1 Perm Federal Research Center of the Ural Branch of The Russian Academy of Sciences, Lab of Ecological \\ Immunology, Institute of Ecology and Genetics of Microorganisms, 614081 Perm, Russia; \\ krasnykh-m@mail.ru (M.B.); timganovavp@gmail.com (V.T.); mantissa7@mail.ru (S.Z.); \\ mraev@iegm.ru (M.R.) \\ 2 Department of Biology, Perm State University, 614068 Perm, Russia; izotrofan@mail.ru (O.B.); \\ lasest1999@gmail.com (S.L.); anast218bio@gmail.com (A.N.) \\ 3 Center for Immunology and Cellular Biotechnology, Immanuel Kant Baltic Federal University, \\ 236016 Kaliningrad, Russia \\ 4 Perm Federal Research Center of the Ural Branch of The Russian Academy of Sciences, Institute of Technical \\ Chemistry, 614013 Perm, Russia; dkiselkov@yandex.ru \\ check for \\ updates \\ Citation: Khramtsov, P.; Burdina, O.; \\ Lazarev, S.; Novokshonova, A.; \\ 5 Lab of Applied Magnetism, M.N. Mikheev Institute of Metal Physics of the UB RAS, \\ 620108 Yekaterinburg, Russia; calamatica@gmail.com \\ 6 Faculty of Biology and Fundamental Medicine, Ural Federal University Named after The First President of \\ Russia B.N. Yeltsin, 620002 Yekaterinburg, Russia \\ * Correspondence: khramtsovpavel@yandex.ru; Tel.: +7-342-280-77-94
} Bochkova, M.; Timganova, V.; Kiselkov, D.; Minin, A.; Zamorina, S.; Rayev, M. Modified Desolvation Method Enables Simple One-Step Synthesis of Gelatin Nanoparticles from Different Gelatin Types with Any Bloom Values. Pharmaceutics 2021, 13, 1537. https://doi.org/ 10.3390/pharmaceutics13101537

Academic Editors: Melgardt de Villiers and Daniel P. Otto

Received: 28 July 2021

Accepted: 18 September 2021

Published: 22 September 2021

Publisher's Note: MDPI stays neutral with regard to jurisdictional claims in published maps and institutional affiliations.

Copyright: (C) 2021 by the authors Licensee MDPI, Basel, Switzerland. This article is an open access article distributed under the terms and conditions of the Creative Commons Attribution (CC BY) license (https:// creativecommons.org/licenses/by/ $4.0 /)$.

\begin{abstract}
Gelatin nanoparticles found numerous applications in drug delivery, bioimaging, immunotherapy, and vaccine development as well as in biotechnology and food science. Synthesis of gelatin nanoparticles is usually made by a two-step desolvation method, which, despite providing stable and homogeneous nanoparticles, has many limitations, namely complex procedure, low yields, and poor reproducibility of the first desolvation step. Herein, we present a modified one-step desolvation method, which enables the quick, simple, and reproducible synthesis of gelatin nanoparticles. Using the proposed method one can prepare gelatin nanoparticles from any type of gelatin with any bloom number, even with the lowest ones, which remains unattainable for the traditional two-step technique. The method relies on quick one-time addition of poor solvent (preferably isopropyl alcohol) to gelatin solution in the absence of stirring. We applied the modified desolvation method to synthesize nanoparticles from porcine, bovine, and fish gelatin with bloom values from 62 to 225 on the hundreds-of-milligram scale. Synthesized nanoparticles had average diameters between 130 and $190 \mathrm{~nm}$ and narrow size distribution. Yields of synthesis were $62-82 \%$ and can be further increased. Gelatin nanoparticles have good colloidal stability and withstand autoclaving. Moreover, they were non-toxic to human immune cells.
\end{abstract}

Keywords: encapsulation; nanoprecipitation; coacervation; manufacturing; yield; nanocarriers; drug delivery

\section{Introduction}

Gelatin is a product of partial hydrolysis of collagen. In the course of gelatin preparation, collagen is pre-treated under acidic or alkaline conditions, which results in obtaining two types of gelatin: type A and type B, respectively. The main sources of gelatin are bovine skin, bovine hides, and cattle and pork bones, whereas fish and poultry gelatins are used to a limited extent [1]. Gelatin from cold-water fish contains a lower percentage of proline and hydroxyproline which are involved in the formation of collagen-like triple helices and therefore has inferior gelation properties in comparison with mammalian gelatins [1,2]. 
Being biocompatible (included in FDA's GRAS list), low-immunogenic, cheap, and commonly available biopolymer gelatin gains popularity in biomedicine, biotechnology, and food science [3]. Conditions in which hydrolysis of collagen is performed affect the size distribution of resulting gelatin molecules. Size distribution of gelatin molecules usually correlates with gel strength which is expressed as a bloom value: the longer the gelatin polypeptide chains the higher the gel strength and bloom value [4].

Gelatin, as well as many other proteins, is used in the form of gelatin nanoparticles. Nanoparticles are small particles with sizes from several nanometers to hundreds of nanometers [5]. Gelatin nanoparticles consist of numerous gelatin molecules which are covalently cross-linked or stabilized by a coating layer [3]. Drug delivery is arguably the scientific field most intensively taking advantage of gelatin nanoparticles. Various drug molecules, DNA, imaging agents (e.g., radiotracers or fluorescent dyes), or nutrients can be physically entrapped in the nanoparticle body or chemically attached to their surface [6]. Tuning of nanoparticles' diameter, coating, and cross-linking degree allows to control circulation time, cellular uptake, as well as drug release rate [6,7]. Gelatin nanoparticles offer a number of unique advantages, namely, they provide improved pharmacokinetics, release profile, and localized delivery of drugs while retaining favorable properties of gelatin including biodegradability along with low cytotoxicity and low immunogenicity [7]. Many reports of gelatin-based nanotherapeutics and nanovaccines were made in the past years [8]. Below some representative examples of successful in vivo applications of gelatin nanoparticles-based nanomedicines are presented.

Application of gelatin nanoparticles allowed the same therapeutic effect with the five-fold lower dose of timolol maleate for glaucoma treatment on a mouse model in comparison with conventional therapy (free timolol maleate) [9]. Gelatin nanoparticles and their aminated counterparts exhibited immunomodulatory efficiency comparable to that of aluminum adjuvants being non-immunogenic by themselves [10]. Pegylated gelatin nanoparticles showed excellent biocompatibility and significantly improved release kinetics and bioavailability of ibuprofen after parenteral administration [11]. Gelatin nanoparticles loaded with immunostimulatory cytosine-phosphate-guanosine oligodeoxynucleotides provided long-term positive effects in horses with asthma and showed higher efficacy in comparison with standard therapy [12]. Antimicrobial gelatin nanoparticles modified with selenium nanoparticles and ruthenium complexes and coated with erythrocyte membranes were tested in vivo on mice. Nanoparticles accumulated in the injury site and provided elimination of methicillin-resistant Staphylococcus aureus; their efficiency was equal to that of vancomycin [13].

Potential applications of gelatin nanoparticles are not limited to therapeutics and vaccine development. Gelatin nanoparticles and microparticles together with molecular gelatin can serve as cheap collagen substitutes imitating extracellular matrix in cell culturing and tissue engineering [14]. Gelatin nanoparticles improve the mechanical properties of bioinks for 3D bioprinting [15,16] and increase circulating tumor cell capture in a microfluidic device [17]. Preparation of Pickering emulsions for food chemistry is another prominent application of gelatin nanoparticles [18].

Desolvation is one of the most popular techniques for gelatin nanoparticle synthesis [3]. This method relies on the addition of poor solvents (usually acetone, alcohols, or acetonitrile) to the aqueous protein solution. Desolvation of gelatin is regularly performed in two steps according to the method described by Coester et al. [19] and further optimized by researchers from the same scientific group $[20,21]$. The first step of desolvation includes the addition of non-solvent to gelatin solution resulting in sedimentation of high-molecular gelatin fractions. Sediment is dissolved in water, then, after the $\mathrm{pH}$ adjustment, repeated addition of non-solvent results in the formation of gelatin nanoparticles. Being a relatively simple and accessible method of synthesis of stable and biocompatible gelatin nanoparticles, two-step desolvation is widely used in various fields. Shortcomings of two-step desolvation are low particle yields, lack of reproducibility of the first desolvation step, and difficult process scale-up [22]. Therefore, numerous efforts were made to develop a more 
straightforward, one-step technique. It has been shown that the presence of low-molecularweight fractions (more than $20 \%$ of fractions with molecular weight less than $65 \mathrm{kDa}$ ) in gelatin preparations leads to the formation of non-stable and polydisperse nanoparticles. These very fractions need to be removed with the first desolvation step [20,21].

Several approaches were proposed to prepare gelatin nanoparticles by the desolvation method in one step. The first approach is to use custom-made or recombinant gelatin lacking low-molecular-weight fractions [21,23]. The disadvantage of this method is limited availability and the high cost of starting material. Commercially available high-bloom gelatin (bloom value of 300) allows to skip the first desolvation step [22], however, resulting nanoparticles tend to aggregate [24]. Vacuum filtration was used to get rid of large molecular weight gelatin and increase the homogeneity of gelatin before desolvation [25]. Shamarekh et al., prepared gelatin enriched with high-molecular-weight fractions from commercial gelatin and used it as a starting material [26]. Despite these last two methods allowing desolvation to be made in one step, they are rather quasi-one-step than true one-step because both of them still require depletion of smaller gelatin molecules.

Surprisingly, several research groups reported the synthesis of uniform gelatin nanoparticles by one-step desolvation without removal of low-molecular-weight fractions [9,27-29]. All these groups used gelatin with bloom 225 or lower, which is expected to be not compatible with the one-step method. Most of these works lack an explanation of why proposed synthesis protocols are effective, however, Ofokansi et al., claimed that neutral $\mathrm{pH}$ facilitated stability and homogeneity of nanoparticles [29].

We revealed that the stirring speed of the gelatin solution dramatically influences the desolvation process. Intensive stirring promotes gelatin aggregation. Using a simple one-step stirring-free approach we previously prepared stable and homogeneous gelatin nanoparticles from gelatin with bloom values as low as 75 [30]. Based on these findings we intended to develop a facile method for nanoparticle preparation from any type of gelatin with any bloom number. Hence, the goals of this work were as follows:

1. To confirm the effect of stirring on desolvation of gelatin;

2. To study the influence of gelatin $\mathrm{pH}$, concentration, and non-solvent type on the size and yield of gelatin nanoparticles;

3. Using optimized conditions to synthesize nanoparticles from porcine, bovine, and fish gelatin with different bloom values (including lowest values available) in a hundreds-of-microgram scale;

4. To study storage stability and colloidal stability of resulting nanoparticles;

5. To load model hydrophobic molecules into gelatin nanoparticles;

6. To assess the effect of sterilization on the integrity of gelatin nanoparticles;

7. To study cytotoxicity of gelatin nanoparticles prepared by modified desolvation method.

\section{Materials and Methods}

\subsection{Materials}

Gelatin B, 75 bloom (lot\# G6650); gelatin B, 225 bloom (lot\# G9382); cold-water fish gelatin (lot\# G7041), gelatin A, 62 bloom (lot\# 48720); gelatin A, 180 bloom (lot\# 48722) BCA assay kit, 1,10-phenanthroline, and boric acid were obtained from Sigma Aldrich (Burlington, MA, USA). Glutaraldehyde (50\%) was obtained from ITW Reagents (Glenview, IL, USA). Trypsin was obtained from Samson-Med (Saint-Petersburg, Russia). Diacoll was obtained from Dia-M (Moscow, Russia). Propidium iodide was obtained from eBioscience (Santa Clara, CA, USA). DMSO was obtained from Tula Pharmaceutical Plant (Tula, Russia). Water for injections was obtained from Solopharm (Saint-Petersburg, Russia). Sodium hydroxide, sodium chloride, sodium hydrogen phosphate, sodium dihydrogen phosphate, sodium bicarbonate, sodium carbonate, glycine were obtained from ITW Reagents (Glenview, IL, USA). Isopropyl alcohol, ethanol, methanol, hydrochloric acid, acetic acid were obtained from Vekton (Saint-Petersburg, Russia). 
4-(4-methylphenyl)-2,4-dioxobutanoic acid was obtained from commercially available reagents by the Claisen condensation [31] and kindly provided by Dr. Ekaterina Khramtsova, department of Organic Chemistry, PSU.

The following instrumentation was used: peristaltic pump, LKB (Upsalla, Sweden), Synergy H1 plate reader, BioTek (Winooski, VT, USA), Multiskan Sky UV-Vis Reader, Thermo (Waltham, MA, USA) and ZetaSizer NanoZS particle analyzer, Malvern (Malvern, UK), CytoFLEX flow cytometer, Beckman Coulter (Brea, CA, USA), SV-10 viscometer, A\&D (Tokyo, Japan). Multipette M4, Eppendorf (Hamburg, Germany) was used for accurate dispensing of viscous gelatin solutions.

\subsection{Preparation of Gelatin Stock Solutions}

Gelatin powder was added to a certain volume of water and incubated at $+40{ }^{\circ} \mathrm{C}$ until a clear solution was obtained. Gelatin solution was aliquoted and stored at $+4{ }^{\circ} \mathrm{C}$. The concentration of gelatin was determined gravimetrically as follows. Gelatin solution $(1 \mathrm{~mL})$ was added to the porcelain crucible and dried to constant weight at subsequently +95 and $+140{ }^{\circ} \mathrm{C}$. Three replicates were done for each sample. The concentration of gelatin nanoparticles was measured in the same way.

\subsection{Synthesis of Gelatin Nanoparticles in Hundred-of-Milligram Scale}

Gelatin A (bloom 62 and 180), gelatin B (bloom 75 and 225), and fish gelatin were diluted in water to $10 \mathrm{mg} / \mathrm{mL}$, then $\mathrm{pH}$ was adjusted to 10 (to 11 for gelatin A and fish gelatin) with $1 \mathrm{M} \mathrm{NaOH}$. One hundred milliliters of the resulting solution were desolvated by $500 \mathrm{~mL}$ of isopropyl alcohol, and the mixture was incubated for $30 \mathrm{~min}$ at $+37^{\circ} \mathrm{C}$ in the water bath. Gelatin solutions and isopropyl alcohol were kept in the water bath at $+37^{\circ} \mathrm{C}$ prior to mixing. Then, $22.5 \mathrm{~mL}$ of $0.8 \%$ glutaraldehyde was quickly added, followed by 30 min long incubation at $+37^{\circ} \mathrm{C}$ in the water bath. Cross-linked nanoparticles were transferred into polycarbonate $85 \mathrm{~mL}$ centrifuge tubes and centrifuged at $15,000 \times g$ for $60 \mathrm{~min}$. Pellets were combined and redispersed in $60 \mathrm{~mL}$ of water with sonication (10-30 s, $60 \%$ amplification, $3 \mathrm{~mm}$ probe, approx. $8 \mathrm{~W}$ ), resulting suspensions were centrifuged at $15,000 \times g$ for $30 \mathrm{~min}$ two more times. After the final centrifugation, $40 \mathrm{~mL}$ of water was added to the pellet, and the resulting suspension was sonicated for $20 \mathrm{~min}(60 \%$ amplification, $6 \mathrm{~mm}$ probe, approx. $25 \mathrm{~W}$ ) on ice. The concentration of nanoparticles was determined by gravimetric analysis.

For fluorescence measurements samples were diluted to $1 \mathrm{mg} / \mathrm{mL}$ with water; then, $100 \mu \mathrm{L}$ of each sample was transferred into the wells of black 96-well plates. The size of nanoparticles was determined by the dynamic light scattering (DLS) technique. For DLS measurements nanoparticles were diluted at 1:375 in water. Hereinafter z-average hydrodynamic diameters (Dh) are given. In order to obtain scanning electron microscopy (SEM) images, nanoparticles were diluted in water to $1 \mu \mathrm{g} / \mathrm{mL}$, dropped at $5 \times 5 \mathrm{~mm}$ silicon wafer, and dried overnight at room temperature. For SEM experiments glycinequenched nanoparticles were taken to reduce the possibility of interaction between free aldehyde groups and amine groups located on the nanoparticles' surface in the course of drying.

\subsection{Preparation of Gelatin Nanoparticles Loaded with Fluorescent Europium Chelates}

Gelatin A (bloom 62 and 180), gelatin B (bloom 75 and 225), and fish gelatin were diluted in water to $10 \mathrm{mg} / \mathrm{mL}$ then $\mathrm{pH}$ was adjusted to 10 (to 11 for gelatin $\mathrm{A}$ and fish gelatin) with $1 \mathrm{M} \mathrm{NaOH}$. Four milliliters of the resulting solution were desolvated by $20 \mathrm{~mL}$ of ethanol containing 4-(4-Methylphenyl)-2,4-dioxobutanoic acid, 1,10-phenanthroline, and europium chloride (concentrations were 180, 60, and $60 \mu \mathrm{M}$, respectively), and the mixture was incubated for $30 \mathrm{~min}$ at $+37^{\circ} \mathrm{C}$ in the thermostat [32]. Solutions containing gelatin and fluorescent complexes were kept on the water bath at $+37^{\circ} \mathrm{C}$ before mixing. Then, $900 \mu \mathrm{L}$ of $0.8 \%$ glutaraldehyde was added, followed by $30 \mathrm{~min}$ long incubation at $+37^{\circ} \mathrm{C}$. Nanoparticles were transferred into polycarbonate $85 \mathrm{~mL}$ centrifuge tubes and centrifuged 
at $15,000 \times g$ for $60 \mathrm{~min}$. Pellet was redispersed in $4 \mathrm{~mL}$ of water with sonication and centrifuged three times at $20,000 \times g$ for $30 \mathrm{~min}$. After each wash pellet was redispersed in water by sonication (10-30 s, $60 \%$ amplification, $3 \mathrm{~mm}$ probe, approx. $8 \mathrm{~W}$ ). Supernatants obtained after the final washing step were collected. For fluorescence measurements 10fold dilutions of nanoparticles in water were prepared; then $100 \mu \mathrm{L}$ of each dilution were transferred into the wells of black 96-well plates.

\subsection{Steam Autoclaving}

Glycine quenching. Before sterilization, $1 \mathrm{M}$ glycine-NaOH buffer $\mathrm{pH} 9.3$ was added to gelatin nanoparticles suspension (1 part of buffer per 9 parts of suspension) and the resulting mixture was incubated for $1 \mathrm{~h}$ at $+37^{\circ} \mathrm{C}$ on a rotator (10 rpm, 360 degrees). Nanoparticles were then washed three times with water by centrifugation at $15,000 \times g$ for $1 \mathrm{~h}$. The concentration of nanoparticles was determined by gravimetric analysis.

Autoclaving. Five milliliters of the resulting nanoparticle suspension were placed in the $15 \mathrm{~mL}$ amber glass vials and autoclaved for $15 \mathrm{~min}$ at $0.5 \mathrm{~atm}$ above atmospheric pressure. Suspensions were cooled at room temperature and stored at $+4{ }^{\circ} \mathrm{C}$. Control nanoparticles were kept at $+4{ }^{\circ} \mathrm{C}$.

Removal of nanoparticle aggregates after autoclaving. A total of $1 \mathrm{~mL}$ of nanoparticle suspension was moved to centrifuge tubes. Nanoparticles were centrifuged at $1000 \times g$ for $10 \mathrm{~min}$. After centrifugation nanoparticle size was measured by DLS. Triple replicates were performed for each measurement.

Characterization. The optical density of nanoparticle suspensions was measured before and after centrifugation. The suspension was diluted in distilled water. The measurement was performed at $600 \mathrm{~nm}$. For DLS measurements nanoparticles were diluted at 1:375 in PBS ( $\mathrm{pH} 7$ ). Zeta potential of autoclaved nanoparticles was measured at $\mathrm{pH} 7$ and ionic strength of $0.06 \mathrm{M}$. Ionic strength was adjusted with $1 \mathrm{M}$ KNO3. The measurements were done with three technical replicates.

\subsection{Assessment of Nanoparticle Stability at Different $\mathrm{pH}$ and High Salt Concentrations}

Stability of nanoparticles at different $\mathrm{pH}$ values. Nanoparticles were diluted to $50 \mu \mathrm{g} / \mathrm{mL}$ in buffer solutions with $\mathrm{pH}$ ranging from 4 to 10 . The following buffers were

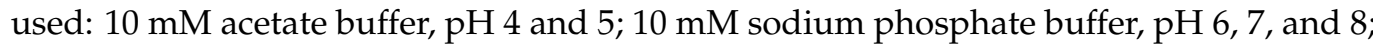
$10 \mathrm{mM}$ borate buffer, $\mathrm{pH} 9$ and 10 . Ionic strength was adjusted to $0.15 \mathrm{M}$ by the addition of $\mathrm{NaCl}$. Nanoparticle size at each $\mathrm{pH}$ was measured immediately by DLS. Three replicates were done for each measurement. Nanoparticle suspensions were stored for 7 days in plastic cuvettes, which were placed in a wet chamber. On days 1 and 7 additional size measurements were performed.

Stability of nanoparticles at different salt concentrations. Nanoparticles were diluted to $50 \mu \mathrm{g} / \mathrm{mL}$ in a phosphate buffer, $\mathrm{pH}$ 7. The ionic strength of the solution was increased to $0.5,1,2$, and $3 \mathrm{M}$ by the addition of $\mathrm{NaCl}$. Nanoparticle size was measured by DLS for each salt concentration. Viscosity of $\mathrm{NaCl}$ solutions was determined with the aid of viscometer and were $1.265 \mathrm{mPa} \cdot \mathrm{s}(3 \mathrm{M} \mathrm{NaCl}), 1.145 \mathrm{mPa} \cdot \mathrm{s}(2 \mathrm{M} \mathrm{NaCl}), 0.992 \mathrm{mPa} \cdot \mathrm{s}(1 \mathrm{M}$ $\mathrm{NaCl})$ and $0.983 \mathrm{mPa} \cdot \mathrm{s}(0.5 \mathrm{M} \mathrm{NaCl})$.

Measurement of nanoparticle zeta potential at different $\mathrm{pH}$. Nanoparticle suspensions were diluted to $50 \mu \mathrm{g} / \mathrm{mL}$ in the following buffers: $10 \mathrm{mM}$ acetate buffer, $\mathrm{pH} 4$ and 5; $10 \mathrm{mM}$ sodium phosphate buffer, $\mathrm{pH} \mathrm{6,7,} \mathrm{and} \mathrm{8;} 10 \mathrm{mM}$ borate buffer, $\mathrm{pH} 9$ and 10. Ionic strength was adjusted to $0.06 \mathrm{M}$ by the addition of KNO3. Three technical replicates were performed for each measurement.

\subsection{Cell Viability Study}

Venous blood was drawn from three healthy volunteers (from 23 to 31 years old) into heparin-contained vacuum tubes. Peripheral blood mononuclear cells (PBMC) were isolated from blood plasma by density gradient centrifugation with Diacoll $(1077 \mathrm{~g} / \mathrm{L}$, Dia-M, Moscow, Russia) at $400 \mathrm{~g}$ for $40 \mathrm{~min}$. Isolated cells were washed with Hanks' 
Balanced Salt solution three times; then, cells were seeded in duplicates into 96-well plates $\left(200 \mu \mathrm{L}\right.$ per well, $1 \times 10^{6}$ cells $/ \mathrm{mL}$ ). Thirty microliters of sterilized (see Section 2.5$)$ gelatin nanoparticles diluted in water for injections (WFI) were added into each well. Final concentrations of gelatin nanoparticles were 1000, 250,62.5, 15.6, and $3.9 \mu \mathrm{g} / \mathrm{mL}$. The negative and positive controls were WFI and 15\% DMSO, respectively [33]. Cells were incubated for $24 \mathrm{~h}$ in a humidified atmosphere in the $\mathrm{CO}_{2}$ incubator $\left(5 \%\right.$ of $\left.\mathrm{CO}_{2},+37^{\circ} \mathrm{C}\right)$, stained with propidium iodide (PI) $(1 \mu \mathrm{g} / \mathrm{mL}, 5 \mu \mathrm{L}$ for $100 \mu \mathrm{L}$ of cell suspension) for one minute, and analyzed by flow cytometry. The percentage of PI- (living) cells was determined for each sample.

Monocytes engulfing particles fluoresce in the emission spectrum of propidium iodide (maximum about $615 \mathrm{~nm}$ ) (Figure S7). Therefore, gates for living (PI-) and dead (PI+) cells were set according to unstained samples and positive/negative controls (Figure S8).

The granularity of nanoparticles-engulfing cells, and, accordingly, the side light scatter (SSC) parameters increases [34]. Therefore, the engulfing of particles by monocytes was determined by the geometric mean of side scattering intensity (Figure S9).

\section{Results and Discussion}

\subsection{Stirring Promotes Nanoparticle Aggregation in the Course of Desolvation}

We revealed that quick one-time addition of non-solvent to aqueous gelatin solution without agitation leads to the formation of monodisperse gelatin nanoparticles. Moreover, in the course of preliminary experiments nanoparticles were successfully prepared from gelatin B with bloom values as low as 75 (molecular weight in the range between 20 and $25 \mathrm{kDa}$ according to manufacturer). This result contradicts conclusions made by other researchers: usually, removal of low-molecular-weight gelatin fractions is necessary to obtain stable and fine nanoparticle suspensions [20,21]. We suggest that stirring of gelatin solution upon addition of non-solvent promotes aggregation of gelatin molecules and, thus, can be completely omitted.

To prove our suggestion, we performed the following experiment. Ethanol was quickly added to gelatin B (75 bloom) solutions (10 and $20 \mathrm{mg} / \mathrm{mL})$. Then, the suspensions were mixed using three regimes: (1) gentle mixing on a rotator (10 rpm); (2) slow vortexing (100 rpm); (3) fast vortexing (2500 rpm). Three individual batches were prepared for each condition. Their size and polydispersity as well as turbidity (absorbance at $600 \mathrm{~nm}$ ) were measured immediately after the synthesis.

At the gelatin concentration of $10 \mathrm{mg} / \mathrm{mL}$, vortexing has little effect and only the highest speed provokes slight growth of size and turbidity. However, vortexing had a dramatic impact when gelatin concentration was doubled: almost $50 \%$ growth of mean size and turbidity at a low speed and severe aggregation at a high speed. At the same time, homogeneous suspensions of gelatin nanoparticles with polydispersity indices lower than 0.1 were formed when mixing was performed on the rotator (Figure 1). We did not perform experiments with higher gelatin concentrations, but recently we successfully prepared gelatin nanoparticles using $30 \mathrm{mg} / \mathrm{mL}$ gelatin solution which was desolvated under short gentle mixing [30].

Obtained results explain why some researchers were able to carry out the one-step synthesis of gelatin nanoparticles without removal of low-weight gelatin fractions [9,27-29,35-37]. They desolvated solutions with low gelatin concentrations (1\% or less) which are not affected by stirring.

At the same time, Geh et al., synthesized monodisperse gelatin nanoparticles from 40 and $50 \mathrm{mg} / \mathrm{mL}$ gelatin solutions by adding acetone under stirring [22]. The authors used commercially available gelatins A and B with bloom values of 300 and mean molecular weights in the range between 400 and $500 \mathrm{kDa}$. Therefore, we suggest that low-molecularweight fractions in gelatin preparations can promote aggregation in the course of desolvation at high total gelatin concentrations (circa $20 \mathrm{mg} / \mathrm{mL}$ and more) when stirring is carried out. A small percentage or absence of low-molecular-weight fractions enables nanoparticle synthesis under stirring. To reinforce previous findings and demonstrate the 
role of stirring and low-molecular-weight gelatin fractions in the desolvation process two more experiments were performed.

A

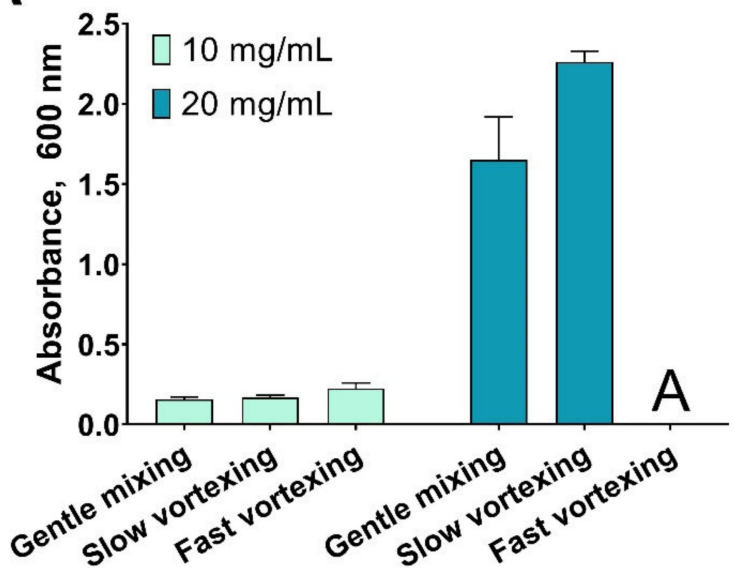

B

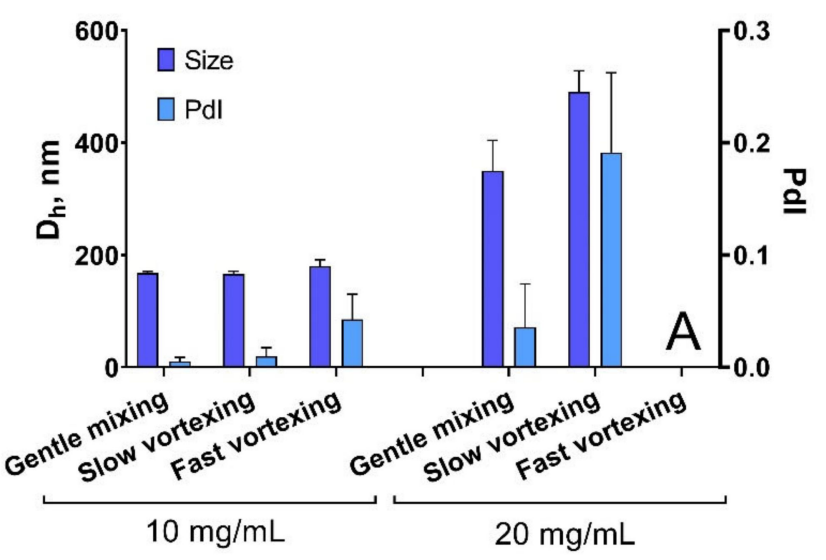

Figure 1. Influence of stirring intensity of the turbidity (A) and size (B) of gelatin nanoparticles. Dh-hydrodynamic diameter; PdI-polydispersity index. Mean values of three individual batches are shown, mean $\pm \mathrm{SD}$. Symbol " $\mathrm{A}$ " stands for aggregation.

Firstly, we desolvated a solution of low-bloom gelatin B (30 mg/mL, pH 9) with ethanol under vigorous stirring and without stirring. One-time addition of non-solvent led to homogeneous suspension of nanoparticles whereas stirring-assisted dropwise addition of ethanol resulted in the formation of gelatin bulk at the bottom of the vial (Figure 2).

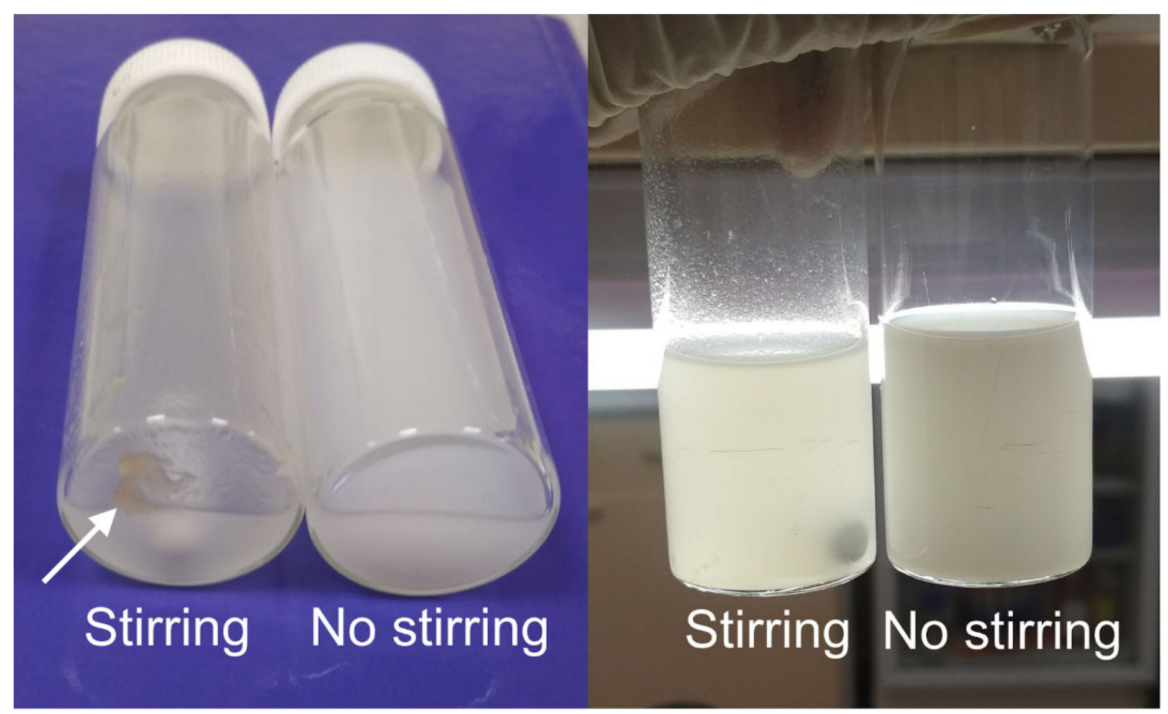

Figure 2. Gelatin nanoparticles prepared from gelatin B, 75 bloom by one-time addition of ethanol without stirring and by dropwise addition of ethanol under stirring. Large gelatin aggregates are labeled with arrows.

In the second experiment, we desolvated with isopropyl alcohol solutions of gelatins A with bloom values of 300 and 62 (both $30 \mathrm{mg} / \mathrm{mL}, \mathrm{pH} 10$ ) or their 3:1, 2:2, or 1:3 mixtures. The total volume of the gelatin solution was $4 \mathrm{~mL}$. Volume fraction of gelatin $\mathrm{A}, 62 \mathrm{bloom}$ varied from $0 \%$ ( $4 \mathrm{~mL}$ of gelatin $\mathrm{A}, 300$ bloom $+0 \mathrm{~mL}$ of gelatin $\mathrm{A}, 62$ bloom) to $100 \%$ ( $0 \mathrm{~mL}$ of gelatin $\mathrm{A}, 300$ bloom $+4 \mathrm{~mL}$ of gelatin $\mathrm{A}, 62$ bloom). As we said before, Geh and colleagues successfully desolvated [22] solution of gelatin A, 300 bloom under stirring. Therefore, this sort of gelatin is suitable for one-step desolvation. Conversely, gelatin A, 
62 bloom has one of the lowest bloom values from commercially available gelatins and should contain mostly low-molecular fractions, being therefore incompatible with the one-step method. By increasing the percentage of low-bloom gelatin in the mixture of two gelatins we studied the role of low-molecular fractions in the desolvation process.

As expected, stirring-assisted desolvation of gelatin mixtures containing $75 \%$ and $100 \%$ of gelatin A, 62 bloom resulted in sedimentation of sticky gelatin mass at the bottom of the vials (Figure S10). On the contrary, no sign of such aggregation was observed in other vials. Visual inspection revealed that more turbid suspensions were obtained when the percentage of low-bloom gelatin exceeded 25\% (Figure S11).

After that, we performed desolvation of low-bloom gelatin A solution under stirring and without stirring, as was done previously for gelatin B, 75 bloom. Again, the homogeneous colloidal solution was obtained in the stirring-free conditions, whereas stirring-assisted desolvation resulted in turbid suspension, containing visible aggregates and gelatin mass at the bottom of the reaction vessel (Figure S12).

Based on the above results, we can conclude that stirring promotes gelatin aggregation in the course of desolvation. Aggregation occurs when low-molecular gelatin fractions are present in sufficient amounts and total gelatin concentration is high (approximately $20 \mathrm{mg} / \mathrm{mL}$ and more). These factors make desolvation of medium- and low-bloom gelatins hardly possible to be made in one step when synthesis is carried out in conventional conditions: dropwise addition of poor solvent under stirring. At the same time, the onetime addition of poor solvent with the following short gentle mixing enables the synthesis of gelatin nanoparticles from gelatin with any bloom number.

Dropwise addition of nonsolvent under vigorous stirring is an inevitable part of protein nanoparticle synthesis by the desolvation method. There are many studies reporting a decrease in size and / or polydispersity of albumin [38], silk fibroin [39], $\alpha$-lactalbumin [40] nanoparticles at higher stirring speeds. The same findings were provided for gelatin nanoparticles by different research groups [41,42]. Conversely, Pei et al. [43] showed that an increase of gelatin concentration in water-ethanol mixture under agitation leads to growth of gelatin nanoparticle size and even to gelation. Removal of low-molecular-weight fractions was not performed in this work as it was aimed at studying gelatin behavior in ethanol-water mixtures rather than the preparation of nanoparticles. Intense shaking provided sedimentation of gelatin 75 bloom in the course of the first desolvation step [44]. There is no contradiction between these reports. Subara and Abdelrady with colleagues removed low-molecular fractions by traditional first desolvation step and studied the effect of stirring speed performing the second desolvation step whereas Pei and colleagues worked with untreated gelatin. Note that Pei et al. [43] used high-bloom gelatins (bloom values of 300 and 320, respectively), which are less susceptible to stirring. We suppose that small amounts of low-molecular fractions in such gelatins can provoke some increase in nanoparticle size, but not aggregation. Additionally, we need to mention a recent paper by Subara and Jaswir in which nanoparticles were synthesized by one-step technique [22] from gelatin hydrolysate comprising small gelatin peptides (about 20 amino acids) [45] Notably, even at concentrations as high as $20 \%$ relatively monodisperse nanoparticles were formed. Therefore, it seems that extremely low gelatin hydrolysate pieces in contrast to low-bloom gelatin allow the preparation of nanoparticles in one step under stirring.

Stirring-free desolvation was applied for the preparation of monodisperse silk fibroin nanoparticles by Seib et al. [46]. The method is to add aqueous silk fibroin solution in acetone in a drop-by-drop manner. However, the same research group reported decreasing the size of silk fibroin nanoparticles when the addition of protein was performed under stirring [39].

We cannot explain why stirring affects gelatin desolvation. Morel et al., studied a mixing-induced aggregation of wheat gluten and proposed that the formation of disulfide and isopeptide bonds as well as hydrophobic interactions can drive aggregation [47]. There are few cysteine residues in gelatin molecules [4], therefore most likely other mechanisms are involved. 


\subsection{Influence of $p H$, Gelatin Concentration, Type, and Volume of Desolvating Agent on the Size and Yield of Gelatin Nanoparticles}

In earlier works, factors affecting the synthesis of gelatin nanoparticles were extensively studied $[20,22,48,49]$. However, the authors of these articles used a conventional technique based on the slow addition of desolvating agents to gelatin solution under stirring. We used a modified desolvation method that relies on the one-time addition of non-solvent to the solution of gelatin. Therefore, we decided to re-evaluate how different factors influence the desolvation outcome. Two sets of experiments were carried out. The first part of the experiments was done using very small volumes of gelatin solution $(200 \mu \mathrm{L})$ and only one type of gelatin. In the second part, five types of gelatin were tested in 20 -fold larger volumes. A detailed description of experiments and results can be found in Supplementary Materials. Below we highlight the key findings on the effects of synthesis conditions on nanoparticle properties.

Isopropyl alcohol was a more effective desolvating agent than ethanol and methanol. It provided homogeneous nanoparticle suspensions with considerably higher yields. Isopropyl alcohol has the lowest polarity index and highest dielectric constant in comparison with methanol and ethanol. It has been reported that the desolvation of $\alpha$-lactalbumin by isopropyl alcohol provided the largest nanoparticles [50]. Moreover, a lower volume of isopropyl alcohol is required to completely desolvate bovine serum albumin in comparison with ethanol [51]. A more complex relationship between properties of non-solvent and its influence on the size of nanoparticles, not limited to the difference of dielectric constants, was demonstrated by Mohammad-Beigi et al. [52]. Pei et al., assumed that differences in alcohols' viscosity can influence the desolvation of gelatin [43]. As a rule, smaller nanoparticles were obtained using ethanol, which is in line with the previous reports [51]. This difference, though, was more distinct at the gelatin concentration of $18 \mathrm{mg} / \mathrm{mL}$. At lower gelatin concentrations, especially at higher $\mathrm{pH}$, ethanol provided a very low degree of gelatin to nanoparticle transformation, which led to unstable DLS results. Larger volumes of alcohols resulted in higher yields due to the lower solubility of gelatin at higher alcohol concentration. Diameters of nanoparticles became lower, probably because the addition of large alcohol volume decreased the final gelatin concentration. Similar trends were observed by Shamarekh et al. [26].

An increase in gelatin concentrations led to the growth of nanoparticle size. This effect was more prominent for gelatins $\mathrm{B}$ and fish gelatin at $\mathrm{pH} 11$. The same relationship was reported by different researchers [22-24,26,43]. Probably, a higher local concentration of gelatin favors the desolvation process, which is also illustrated by higher particle yields at the higher protein concentration [26]. However, other factors, such as gelatin solution viscosity can also impact desolvation results [43].

Higher yields were observed for the same ethanol volume for gelatin $B$ with bloom values of 225 in comparison with gelatin B bloom 75 which is in line with the results obtained by Nixon et al. [53], who showed that lower ethanol volume is necessary to initiate coacervation of gelatins with higher bloom numbers. Interestingly, an opposite relationship was observed for isopropyl alcohol.

Electrostatic repulsion of gelatin molecules and $\mathrm{pH}$-dependent degree of molecule hydration influence both size and yield of nanoparticles as was shown by many researchers $[22,29,54,55]$. Generally, higher $\mathrm{pH}$ values (far away from gelatin isoelectric point) resulted in lower yields and smaller particles.

Obtained results demonstrate that control over synthesis parameters enables tuning of nanoparticles' properties and process yield.

\subsection{Synthesis of Nanoparticles from Different Gelatins in Hundreds-of-Milligram-Scale}

In order to demonstrate the scope of the modified desolvation method, we synthesized nanoparticles from various types of gelatin with different bloom numbers. On the basis of optimization experiments, we decided to desolvate gelatins with isopropyl alcohol to obtain 
high yields of nanoparticles. We intended to prepare nanoparticles with hydrodynamic diameters less than $200 \mathrm{~nm}$, hence $10 \mathrm{mg} / \mathrm{mL}$ gelatin solutions with high $\mathrm{pH}$ were used.

Scalability is an essential part of nanoparticle products implementation. Ideally, the synthesis procedure should be not only scalable but also reproducible, providing small batch-to-batch variability [56]. Optimization experiments were done using rather small portions of gelatin (less than $80 \mathrm{mg}$ ). Here we performed the hundreds-of-milligram-scale synthesis of gelatin nanoparticles by modified desolation method.

We synthesized nanoparticles from porcine, bovine, and fish gelatin with different bloom numbers including the lowest available (62 and 75). The total initial amount of gelatin was $1000 \mathrm{mg}$, three batches were synthesized for each kind of gelatin. Each batch had an ID, indicating the type of gelatin source and number of replication, e.g., "B225-2". Key steps of the synthesis procedure are presented in Figure 3. Isopropyl alcohol was added to gelatin solution, the resulting suspension of gelatin nanoparticles was kept in the water bath for $30 \mathrm{~min}$, then nanoparticles were stabilized by glutaraldehyde, washed by centrifugation, concentrated, and sonicated. Properties of synthesized nanoparticle batches are summarized in Table 1 and Figure 4. In total, we confirmed that the modified desolvation method enables nanoparticle synthesis from different gelatin types with various bloom numbers.
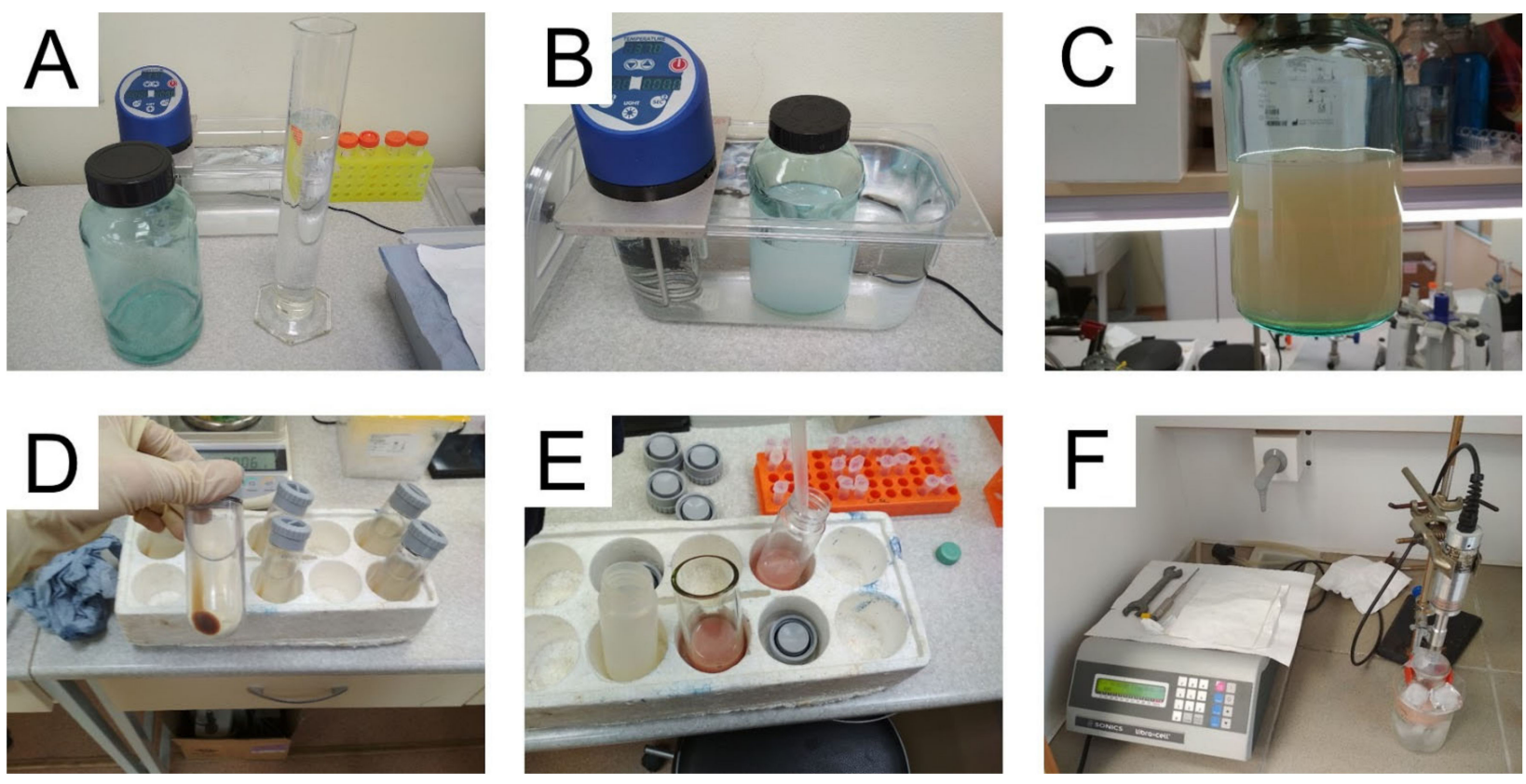

Figure 3. Stages of gelatin nanoparticle synthesis. (A) Solution of gelatin and isopropyl alcohol before mixing; (B) incubation of nanoparticles at the water bath; (C) nanoparticle suspension appearance after glutaraldehyde addition; (D) sediment of nanoparticles after centrifugation; (E) combining of washed gelatin nanoparticles prior to final sonication; (F) sonication of gelatin nanoparticles on ice. 
Table 1. Properties of gelatin nanoparticles prepared from different gelatins.

\begin{tabular}{cccccccc}
\hline Batch ID & $\begin{array}{c}\text { Suspension } \\
\text { Volume, } \mathbf{m L}\end{array}$ & $\begin{array}{c}\text { Concentration, } \\
\mathbf{m g} / \mathbf{m L}\end{array}$ & $\begin{array}{c}\text { Total Dry Weight of } \\
\text { Nanoparticles, } \mathbf{m g}\end{array}$ & $\begin{array}{c}\text { Yield, } \\
\mathbf{\%}\end{array}$ & $\mathbf{D}_{\mathbf{h}, \mathbf{n m}^{\mathbf{1}}}$ & $\mathbf{P d I}^{\mathbf{2}}$ & $\begin{array}{c}\text { Zeta Potential, } \\
\mathbf{m V}\end{array}$ \\
\hline B75-1 & 45 & 18.0 & 810.0 & 81.0 & $189 \pm 8^{3}$ & $0.014 \pm 0.007$ & $-11.5 \pm 0.6$ \\
B75-2 & 46 & 17.7 & 815.6 & 81.6 & $171 \pm 8$ & $0.030 \pm 0.012$ & $-10.2 \pm 0.6$ \\
B75-3 & 44 & 18.2 & 800.8 & 80.1 & $165 \pm 4$ & $0.033 \pm 0.029$ & $-10.7 \pm 0.6$ \\
B225-1 & 45 & 13.8 & 621.0 & 62.1 & $133 \pm 4$ & $0.069 \pm 0.022$ & $-11.5 \pm 0.5$ \\
B225-2 & 45 & 14.4 & 646.2 & 64.6 & $139 \pm 6$ & $0.094 \pm 0.034$ & $-10.9 \pm 0.3$ \\
B225-3 & 45 & 15.2 & 685.4 & 68.5 & $143 \pm 5$ & $0.127 \pm 0.065$ & $-11.1 \pm 0.6$ \\
FISH-1 & 46 & 17.8 & 817.0 & 81.7 & $164 \pm 7$ & $0.114 \pm 0.047$ & $-9.0 \pm 1.0$ \\
FISH-2 & 46 & 16.9 & 778.8 & 77.9 & $156 \pm 6$ & $0.094 \pm 0.052$ & $-9.4 \pm 0.3$ \\
FISH-3 & 46 & 16.9 & 775.6 & 77.6 & $151 \pm 3$ & $0.107 \pm 0.029$ & $-7.1 \pm 0.8$ \\
A62-1 & 46 & 16.9 & 777.4 & 77.7 & $157 \pm 8$ & $0.054 \pm 0.012$ & $-7.9 \pm 0.9$ \\
A62-2 & 45 & 16.4 & 738.0 & 73.8 & $148 \pm 4$ & $0.064 \pm 0.012$ & $-7.8 \pm 1.3$ \\
A62-3 & 45 & 14.5 & 652.5 & 65.3 & $151 \pm 4$ & $0.052 \pm 0.004$ & $-7.5 \pm 0.4$ \\
A180-1 & 45 & 15.8 & 711.0 & 71.1 & $144 \pm 3$ & $0.088 \pm 0.023$ & $-7.1 \pm 0.7$ \\
A180-2 & 46 & 17.0 & 782.0 & 78.2 & $142 \pm 2$ & $0.060 \pm 0.014$ & $-6.9 \pm 0.7$ \\
A180-3 & 45 & 17.1 & 769.5 & 77.0 & $145 \pm 4$ & $0.087 \pm 0.036$ & $-7.4 \pm 0.6$ \\
\hline
\end{tabular}

${ }^{1}$ Hydrodynamic diameter. ${ }^{2}$ Polydispersity index. ${ }^{3}$ Mean of three technical replicates \pm standard deviation.

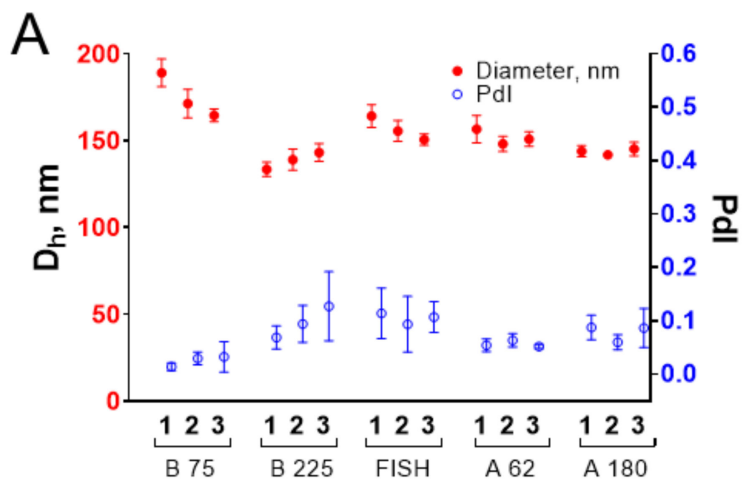

B
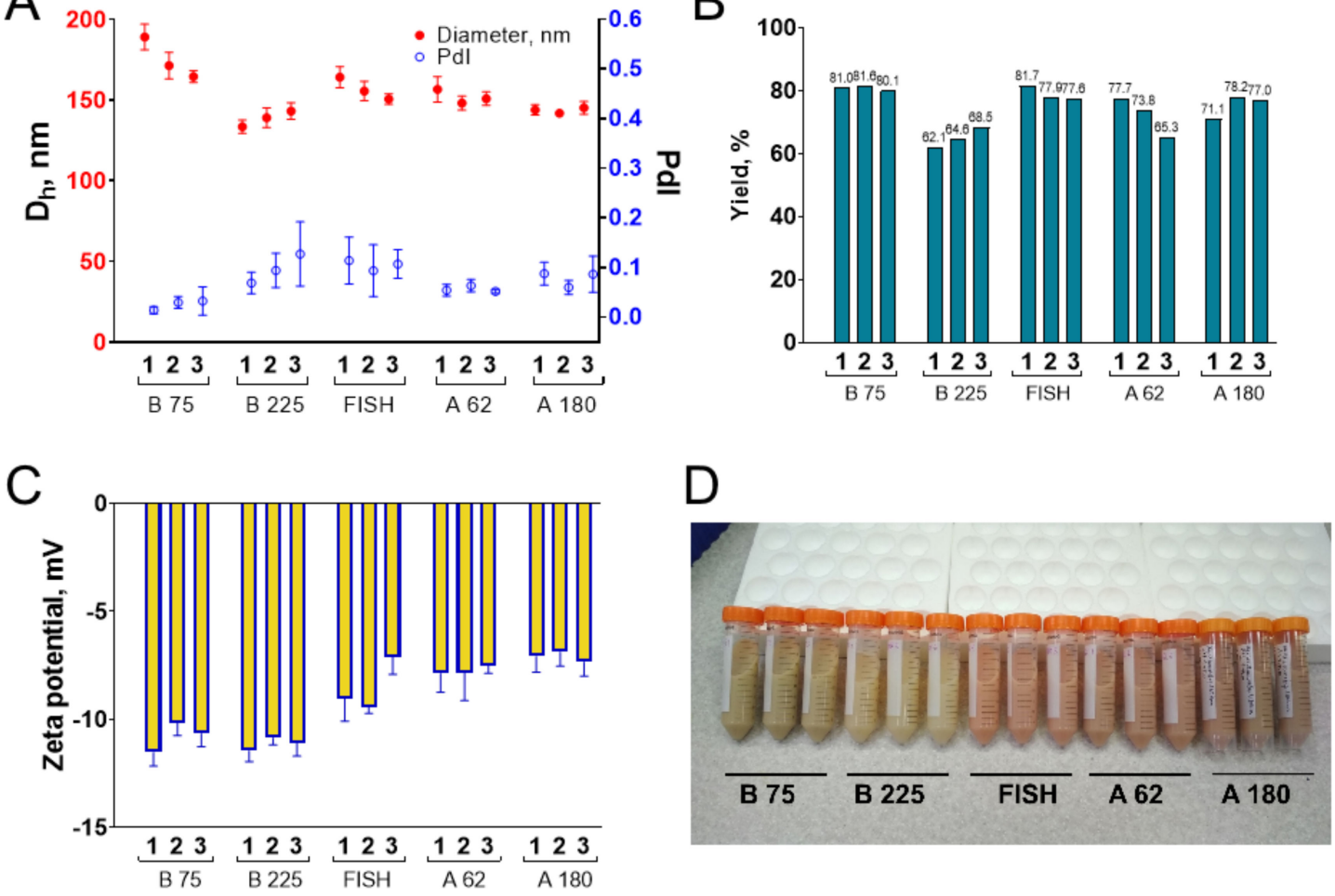

Figure 4. Properties of gelatin nanoparticles prepared from various gelatins in the hundreds-of-milligram scale: (A) size and polydispersity index; (B) yield; (C) zeta potential (at a pH 7); (D) the appearance of gelatin nanoparticle suspensions. Numbers 1, 2, and 3 denote the batch numbers. Dh—hydrodynamic diameter; PdI-polydispersity index. Mean values of three technical replicates are shown, mean $\pm \mathrm{SD}$.

\subsubsection{Size, Zeta Potential, and Shape of Nanoparticles}

The hydrodynamic diameter of most nanoparticles was between 130 and $160 \mathrm{~nm}$. The lowest nanoparticles were prepared from gelatin B, 225 bloom. We assessed the reproducibility of nanoparticle synthesis by calculating coefficients of variation (CV) for each type of gelatin and comparing it with available literature data. Reproducibility was 
the lowest for nanoparticles prepared from gelatin $\mathrm{B}, 75$ bloom $(\mathrm{CV}=7.2 \%)$, whereas for other gelatin types CVs were from $1.2 \%$ to $4.4 \%$. Reproducibility of the preparation of recombinant human serum albumin nanoparticles by desolvation method was studied by Langer et al. [57]. Three batches were prepared; CV was 9.1\% [57]. Gelatin nanoparticles of different sizes prepared by optimized two-step desolvation were reported by Dr. Claus Zwiorek [20]. Six batches were synthesized for each type of gelatin nanoparticles; coefficients of variation were $3.4 \%$ (mean size is $300 \mathrm{~nm}), 1.4 \%(150 \mathrm{~nm})$, and $13.2 \%$ $(100 \mathrm{~nm})$. Thus, the modified desolvation method enables the reproducible synthesis of gelatin nanoparticles. Polydispersity indices were lower than 0.2 for all batches and lower than 0.1 for most batches, indicating that synthesized nanoparticles had homogeneous size distribution.

The zeta potential of nanoparticles was measured in a neutral phosphate buffer, $\mathrm{pH} 7$. According to information from the manufacturer, the isoelectric point is 4.7-5.3 for gelatins B, 7.0-9.5 for gelatins A, and 6.0 for fish gelatin. Nanoparticles prepared from gelatin B had the lowest zeta potential of about $-11 \mathrm{mV}$, whereas nanoparticles made from fish gelatin and gelatin A had more positive zeta potential: from -7 to $-9 \mathrm{mV}$. Notably, the zeta potential of gelatin nanoparticles is much lower than the conditional stability threshold of $\pm 30 \mathrm{mV}$ [58], indicating that forces other than electrostatic repulsion provide their colloidal stability, which is confirmed by the results of their detailed colloidal stability study (see Section 3.4).

Scanning electron microscopy and transmission electron microscopy (TEM) showed that nanoparticles prepared from all types of gelatin had a round shape (Figure 5). The insufficient quality of SEM photographs did not allow us to measure their sizes. Nevertheless, a visual assessment of the photos demonstrated that the sizes of most of the particles are in the range of 100-200 $\mathrm{nm}$, which coincides with the DLS results. The mean diameter of B75-1 obtained by TEM was lower $(100 \pm 21 \mathrm{~nm})$ than the hydrodynamic diameter (Figure S13), which is explained by the well-known tendency of DLS to overestimate the size of nanoparticles. Microscopy demonstrated the presence of large nanoparticles (they can be seen in Figure 5B) and some amount of aggregates, however, in general nanoparticles were homogeneous.
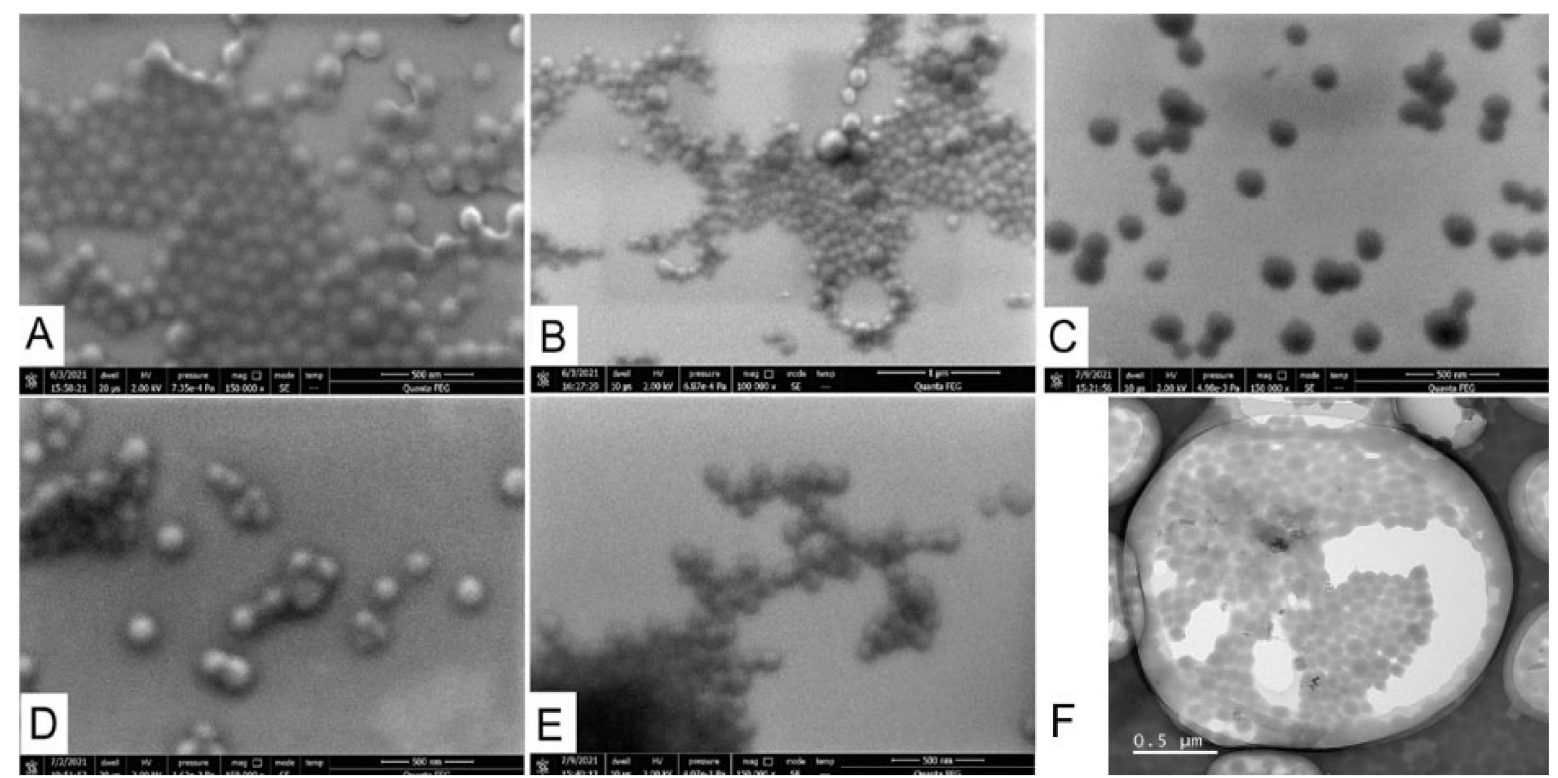

Figure 5. SEM (A-E) and TEM (F) images of gelatin nanoparticles. (A,F) B75-1; (B) B225-1; (C) FISH-1; (D) A62-1; (E) A180-1. Scale bars are $500 \mathrm{~nm}(\mathbf{A}, \mathbf{C}-\mathbf{F})$ or $1000 \mathrm{~nm}(\mathbf{B})$. 


\subsubsection{Yield}

We obtained stable aqueous suspensions of gelatin nanoparticles having volumes of $44-46 \mathrm{~mL}$ and containing from 13.8 to 18.2 milligrams of nanoparticles per milliliter. Therefore, the method allows the preparation of $600-800 \mathrm{mg}$ of nanoparticles in $6-7 \mathrm{~h}$. This value can be increased by the application of larger reagent volumes or by changing the synthesis conditions: decreasing the $\mathrm{pH}$, increasing the gelatin concentration, and so on (see Supplementary Materials). The yield of synthesis (degree of gelatin-to-nanoparticles conversion) was between 62 and $82 \%$, which is higher than reported for two-step and one-step desolvation: $1.5-62 \%$ (Table S1). However, in special conditions yields of conventional one- and two-step desolvation procedures can reach $70-80 \%[22,48,59]$. For the nanoprecipitation method yields as high as $90 \pm 5 \%$ were reported [60], however, lower yields were obtained in other works (Table S1). Based on optimization experiments, we claim that yields up to $95 \%$ can be reached with the aid of the modified desolvation method due to the high desolvating efficiency of isopropyl alcohol.

In the desolvation technique, there is sometimes a trade-off between the yield and size of nanoparticles. Conditions that favor protein desolvation provide better yields, but the larger size of nanoparticles. An increase in yield can be achieved by lowering the $\mathrm{pH}$ or by an increase of added alcohol volume. The second approach enables higher yields and even lower sizes, but at the expense of reaction volume increase. In this work, we synthesized gelatin nanoparticles at high $\mathrm{pH}$ (10 for gelatin $\mathrm{B}$ and 11 for fish gelatin and gelatin $\mathrm{A})$, besides non-solvent to gelatin volume ratio was 5 . Using lower $\mathrm{pH}$ and/or larger ratios, higher yields could be achieved.

One more thing that needs to be explained is lower yields obtained for B225 batches. We think that losses of nanoparticles during washing steps can be the reason. Nanoparticles B225-1/2/3 had the lowest diameters and required more time to complete sedimentation. When decantation of the supernatant was performed, the loose part of the sediment was removed. This was observed for all batches but in the case of B225-1/2/3 it was the most pronounced.

\subsubsection{Absorbance and Fluorescence Spectra of Gelatin Nanoparticles}

Protein nanoparticles cross-linked with glutaraldehyde emit fluorescence when excited by UV or visible light $[30,61]$. Autofluorescence of gelatin nanoparticles can be explained by the presence of $\mathrm{C}=\mathrm{C}$ (resulting from glutaraldehyde polymerization) and $\mathrm{C}=\mathrm{N}$ bonds (in the Shiff bases) in nanoparticles' structure [62]. Fluorescent properties of gelatin nanoparticles can be utilized in bioimaging and biosensing [61]. Moreover, intrinsic fluorescence of nanomaterials could be used to measure their cellular uptake $[63,64]$ and underlines nanoparticle interference with various fluorescent techniques (an example of such interference can be found in Section 3.8). Given that gelatin nanoparticles do not have distinct absorbance peaks (Figure S14A), we recorded the fluorescence spectra of nanoparticles at excitation wavelengths from 260 to $560 \mathrm{~nm}$. Nanoparticles prepared from all gelatins possess broad fluorescent peaks, which are red-shifted with the increase of excitation wavelength (Figures S14B and S15). Further, we used fluorescent properties of gelatin nanoparticles to assess the change of their structure after the sterilization procedure (see Section 3.7).

The color of gelatin nanoparticles depended on synthesis conditions. Desolvation by ethanol at any $\mathrm{pH}$ values or by isopropyl alcohol at $\mathrm{pH}$ less than 11 resulted in yellowish suspension. Nanoparticles prepared at $\mathrm{pH} 11$ using isopropyl alcohol were reddish, indicating possible differences in the chemical structure.

\subsection{Stability of Nanoparticles at Various $\mathrm{pH}$ and Salt Concentrations}

Colloidal stability of nanoparticles at various $\mathrm{pH}$ and in solutions with high ionic strength is highly desirable for practical applications. Conjugation of nanoparticles with different molecules, including recognition molecules (e.g., monoclonal antibodies) as well as a surface modification with stealth or protecting polymers, are usually performed at $\mathrm{pH}$ 
and salt concentration, which are optimal for a specific technique. Therefore, we tested the colloidal stability of nanoparticles in buffers with $\mathrm{pH}$ ranging from 4 to 10 measuring their size by DLS immediately after addition to the buffer, then at days 1 and 7. Gelatin nanoparticles prepared from gelatin B, fish gelatin, and gelatin A 180 bloom were stable for one week in all buffers (Figure 6). A slight increase of size and polydispersity was, though, observed in several samples. In these samples usually, one of three technical replicates indicated the presence of aggregates, whereas other replicates showed homogeneous size distribution. Most likely, insignificant aggregation took place; however, most of the particles were in the non-aggregated state. These results coincide with literature data, indicating that the gelatin shell provides good colloidal stability in the wide range of $\mathrm{pH}$ values [65].
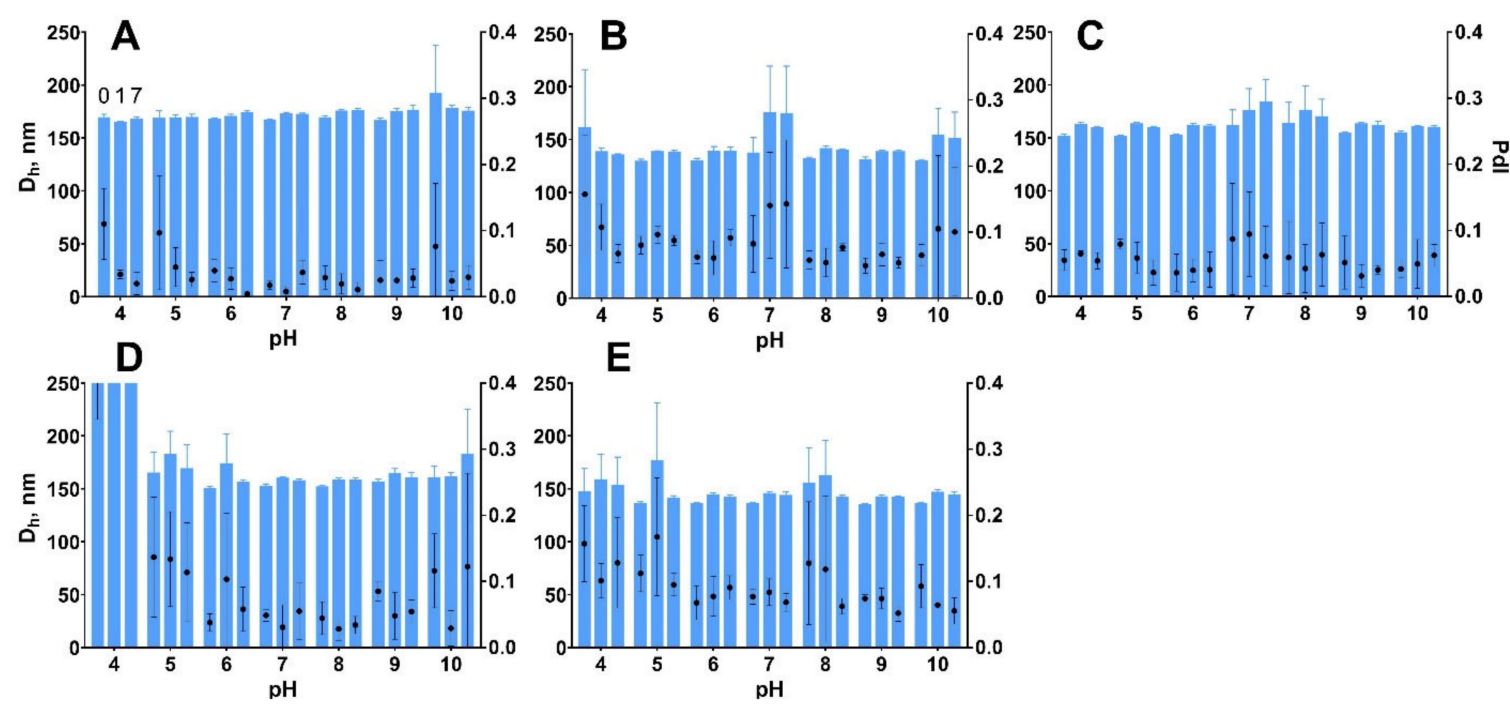

Figure 6. Colloidal stability of gelatin nanoparticles at different pH values. (A) B75-1; (B) B225-1; (C) FISH-1; (D) A62-1; (E) A180-1. Dh-hydrodynamic diameter; PdI-polydispersity index. Mean values of three technical replicates are shown, mean \pm SD. Measurements were performed on days 0,1 , and 7 .

Nanoparticles prepared from gelatin A with a bloom value of 62 were the only type of nanoparticles for which pronounced aggregation was observed (Figure 6D). These nanoparticles quickly aggregated being exposed to $\mathrm{pH} 4$, but not in other buffers. The relationship between zeta potential and $\mathrm{pH}$ was different for nanoparticles prepared from various types of gelatin (Figure S16). Nanoparticles B75 and B225 had more negative zeta potential at a $\mathrm{pH}$ range from 5 to 9 which is explained by the difference in isoelectric points between gelatins [49].

High salt concentrations had no significant effect on the size of gelatin nanoparticles. It was previously shown that low $(7-50 \mathrm{mM})$, but not high $(300 \mathrm{mM}) \mathrm{NaCl}$ concentrations promote aggregation of gelatin nanoparticles [66]. We did not observe any signs of nanoparticle aggregation in presence of salts (Figure S17).

We should note that the concentration of nanoparticles was as low as $50 \mu \mathrm{g} / \mathrm{mL}$. Perhaps, more pronounced aggregation could be detected at higher nanoparticle concentrations. For nanoparticles A180-1/2/3 we detected aggregation at a low nanoparticle concentration (lower than $80 \mu \mathrm{g} / \mathrm{mL}$ ) in water, but not in phosphate buffer. Glycine treatment stabilized these nanoparticles even at low concentrations. We cannot explain this phenomenon, moreover, it was not observed for nanoparticles prepared from other types of gelatin.

\subsection{Storage Stability}

The stability of nanoparticles upon storage is necessary for their practical application in any field. For end users concentrated aqueous suspension is, perhaps, the most convenient 
form of nanoparticle preparations. We studied the size and structural integrity of gelatin nanoparticles prepared using a modified desolvation method after 4 weeks of storage in water at $+4{ }^{\circ} \mathrm{C}$. Surprisingly, a decrease in hydrodynamic diameter and polydispersity indices were detected for all batches (Figure 7). Moreover, after 4 weeks of storage batchto-batch variability of nanoparticle sizes also became lower. These unexpected results contradict the data reported in the literature. Previous works reported no change or growth of nanoparticle diameter $[19,67]$. The decrease of hydrodynamic diameter of gelatin nanoparticles stored in the lyophilized state was observed and explained by incomplete rehydration [68], however, in our study nanoparticles were stored in water and did not change hydration state. We supposed that partial dissolution of nanoparticles could occur. The concentration of free protein in nanoparticle suspension, as well as turbidity of nanoparticle suspension, were monitored (Figure S18). Turbidity can reflect both the change of nanoparticle size and their dissolution. For almost all nanoparticles, a decrease in suspension turbidity was observed. The concentration of free gelatin was also higher on the 28 th day. However, the relative amount of free protein did not exceed $1 \%$ of total gelatin.

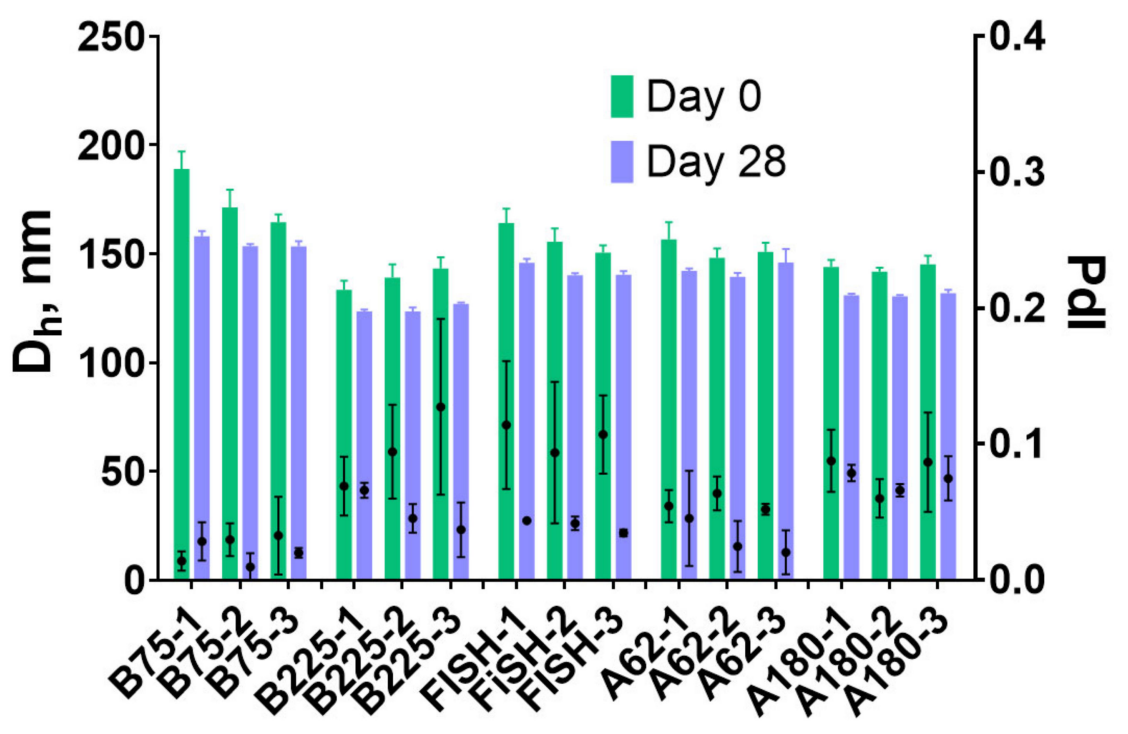

Figure 7. Size (Dh) and polydispersity index (PdI) of gelatin nanoparticles after the synthesis and in 28 days. Mean values of three technical replicates are shown, mean $\pm \mathrm{SD}$.

It can be assumed that the storage of gelatin nanoparticles is accompanied by partial disintegration. Between-batch size variability after 4 weeks of storage became lower, indicating that this process affects larger nanoparticles and aggregates. The percentage of free gelatin was quantified by centrifugation of nanoparticles at 20,000 $\times g$ and measurement of protein in supernatant. Mentioned speed is not high enough to pellet small nanoparticles (say, 10-20 nm). Therefore, the slight increase of protein concentration in supernatants can be explained by both the release of single gelatin molecules and the decomposition of larger nanoparticles into smaller ones. The presence of a certain amount of smaller nanoparticles (in contrast to larger nanoparticles) cannot affect the results of DLS measurements, because their light scattering ability is too small. Taking into account that the degree of turbidity decrease did not correlate with the degree of the particle diameter decrease, we assume that partial decomposition of nanoparticles into smaller nanoparticles took place.

Literature data suggest that glutaraldehyde cross-linking produces stable gelatin nanoparticles $[19,67]$, however, in most cases, only particle size but not other properties were assessed. We examined the storage stability of non-sterile nanoparticle preparations, which were stored in deionized water without any preliminary physical or chemical treatment. Therefore, bacterial contamination and protease activity could play a role in nanoparticle degradation. Undoubtedly, further study of nanoparticle stability needs to be 
conducted. However, even if nanoparticles are unstable in suspension, there are optimized methods of their storage in freeze-dried conditions [68].

\subsection{Loading of Gelatin Nanoparticles with Fluorescent Complex}

Biomedical and biotechnological applications of gelatin nanoparticles require them to be loaded with a wide spectrum of therapeutic and imaging agents: small molecules, nanomaterials, and polymers. We tested whether the modified desolvation method is appropriate for incorporation of model hydrophobic substance: fluorescent complex containing europium ion and two chelating ligands, namely 1,10-phenanthroline and 4-(4Methylphenyl)-2,4-dioxobutanoic acid. Europium and ligands were dissolved in ethanol; then gelatins of each type were desolvated with the resulting solution. Ethanol was chosen because of the insufficient solubility of fluorescent complexes in isopropyl alcohol. Europium complexes possess bright fluorescence facilitating confirmation of successful loading, besides, they are insoluble in water resembling small molecules used in drug delivery. Moreover, the long-living and large-Stokes-shift fluorescence of europium complexes are easily distinguishable from the inherent fluorescence of gelatin nanoparticles.

Nanoparticles synthesized from all the gelatin types were loaded with fluorescent complexes (Figure 8). A narrow peak (600-630 nm) of europium emission was detected in suspensions of purified gelatin nanoparticles after desolvation with an ethanol solution containing fluorescent complexes (Figure S19) but was not detected in bare gelatin nanoparticles (Figure 8). Supernatants obtained during the purification of fluorescent gelatin nanoparticles displayed weak fluorescence, which, nevertheless, was negligible in comparison with that of nanoparticles. Therefore, europium complexes were associated with gelatin nanoparticles, however, we did not study whether they were located on the surface or were embedded in the nanoparticle body. Weak fluorescence in supernatants was most likely due to the leakage of fluorescent complexes induced by ultrasound treatment which accompanied nanoparticle purification.

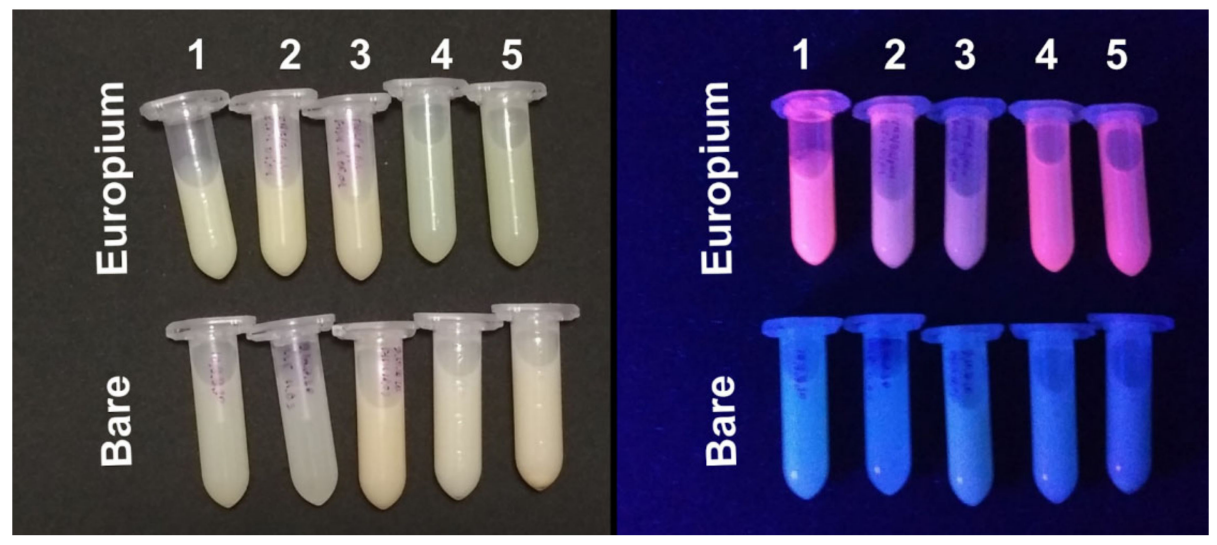

Figure 8. Gelatin nanoparticles containing and not containing europium complexes at daylight (left) and in UV light (360 nm, right). Nanoparticles were prepared from gelatin: $1-\mathrm{B}, 75$ bloom; 2-B, 225 bloom; 3-FISH; 4-A, 62 bloom; 5-A, 180 bloom.

We should note that sub-micro particles and microparticles rather than nanoparticles were synthesized from fish gelatin and type A gelatins (Figure 9A). Type B gelatin 75 bloom yielded the highest quality nanoparticles with the lowest polydispersity indices (Figure 9). The mean diameter of these nanoparticles was $115 \pm 23 \mathrm{~nm}$ (Figure S20), being slightly larger than unloaded ones. 


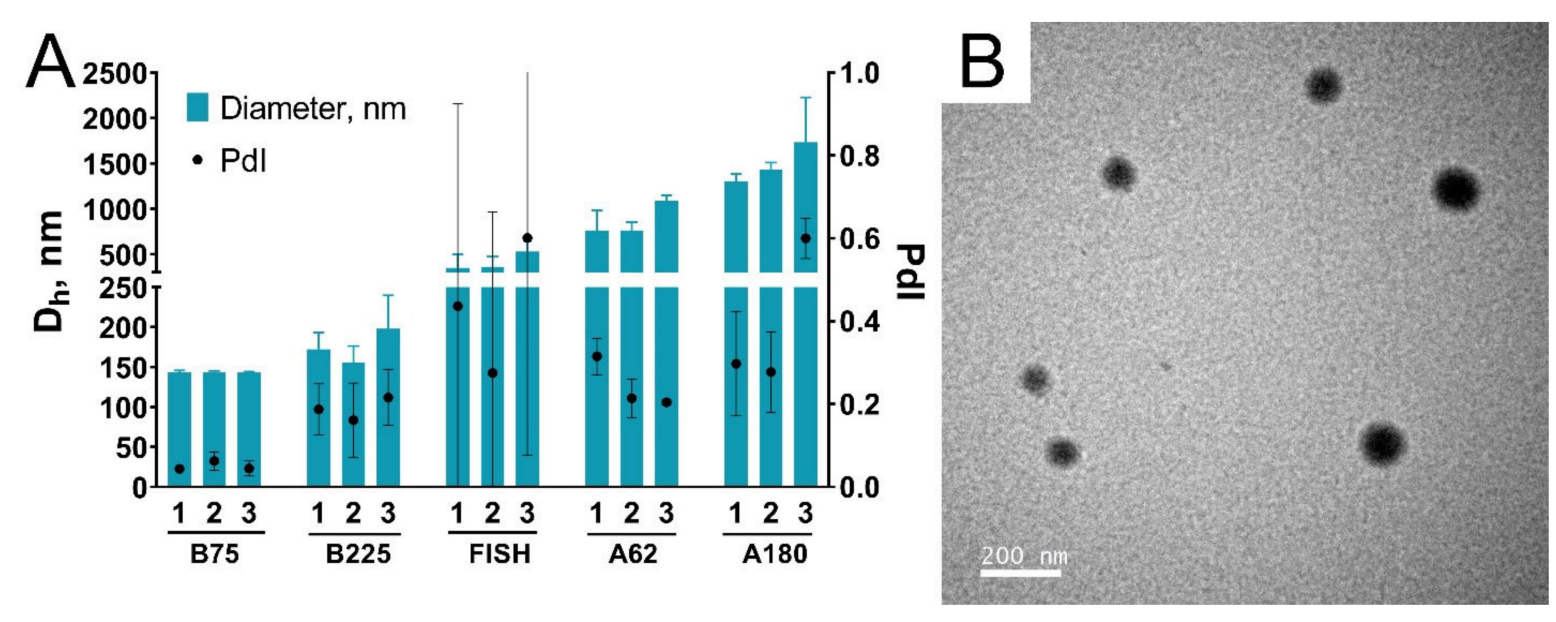

Figure 9. Size of gelatin nanoparticles loaded with fluorescent europium complexes (A) and TEM image of europium-loaded nanoparticles prepared from gelatin B 75 bloom (B). Dh—hydrodynamic diameter; PdI-polydispersity index. Mean values of three technical replicates are shown, mean \pm SD. The scale bar is $200 \mathrm{~nm}$.

In this work, we did not optimize loading conditions, only a couple of preliminary experiments were carried out with type B gelatins. We suppose that proper optimization can enable the preparation of 100-200 nm sized nanoparticles loaded with hydrophobic molecules from any type of gelatin.

\subsection{Sterilization of Gelatin Nanoparticles}

Microbial contamination of gelatin nanoparticles is undesirable for almost all applications. The presence of microorganisms or their fragments in the nanoparticle preparations could pose a risk for patients, besides, microbial enzymes can destroy nanoparticles and decrease their shelf-life [69]. Geh et al., reported that autoclave sterilization of gelatin nanoparticles leads to the partial release of gelatin molecules and slight nanoparticle growth, whereas the higher cross-linking degrees and milder autoclaving conditions make nanoparticles less sensitive to thermal degradation [68]. At the same time, Ma et al., successfully autoclaved bovine serum albumin nanoparticles prepared by the desolvation method [70]. Based on these results we decided to sterilize gelatin nanoparticles by autoclaving in the mildest conditions: $15 \mathrm{~min}$ at $0.5 \mathrm{~atm}$ above atmospheric pressure. Control (non-autoclaved) nanoparticles were kept at $+4{ }^{\circ} \mathrm{C}$.

In autoclaving experiments, we used gelatin nanoparticles treated with glycine. Although we added an excess of glutaraldehyde in relation to the number of primary amines, a small portion of unreacted amino groups can remain on the outer surface of nanoparticles [59]. Glycine quenched free surface carbonyl groups and supposedly decreased the probability of their reaction with remaining primary amines. Unfortunately, glycine treatment led to unstable DLS measurements. DLS results were different even between technical replicates: for some samples, one or two of three measurements indicated the presence of aggregates or increase of average diameter, whereas other measurements showed narrow distribution. This feature of the DLS method was previously well illustrated by Langevin and co-authors [71]. They put $\mathrm{TiO}_{2}$ nanoparticles in a buffer with high ionic strength and measured the diameter of nanoparticles by DLS and differential centrifugal sedimentation, which is much less sensitive to the presence of aggregates than DLS. A large number of aggregates was detected by DLS, on the contrary, differential centrifugal sedimentation showed that the agglomeration degree was not significant.

To obtain more stable DLS results we centrifuged autoclaved nanoparticles for $10 \mathrm{~min}$ at $1000 \times g$ before the size measurements (Figure 10). We suppose that slight aggregation occurred, however, most of the particles withstood sterilization and kept their original size because no visible signs of aggregation were detected (Figure S21). The presence of aggregates and free gelatin may be incompatible with applications, which require very 
homogeneous nanoparticle preparations (e.g., drug delivery). However, autoclaving may be a good and simple choice in the fields with less strict requirements to size distribution. Aggregates can be quickly removed by low-speed centrifugation using commonly available sterile centrifuge tubes.

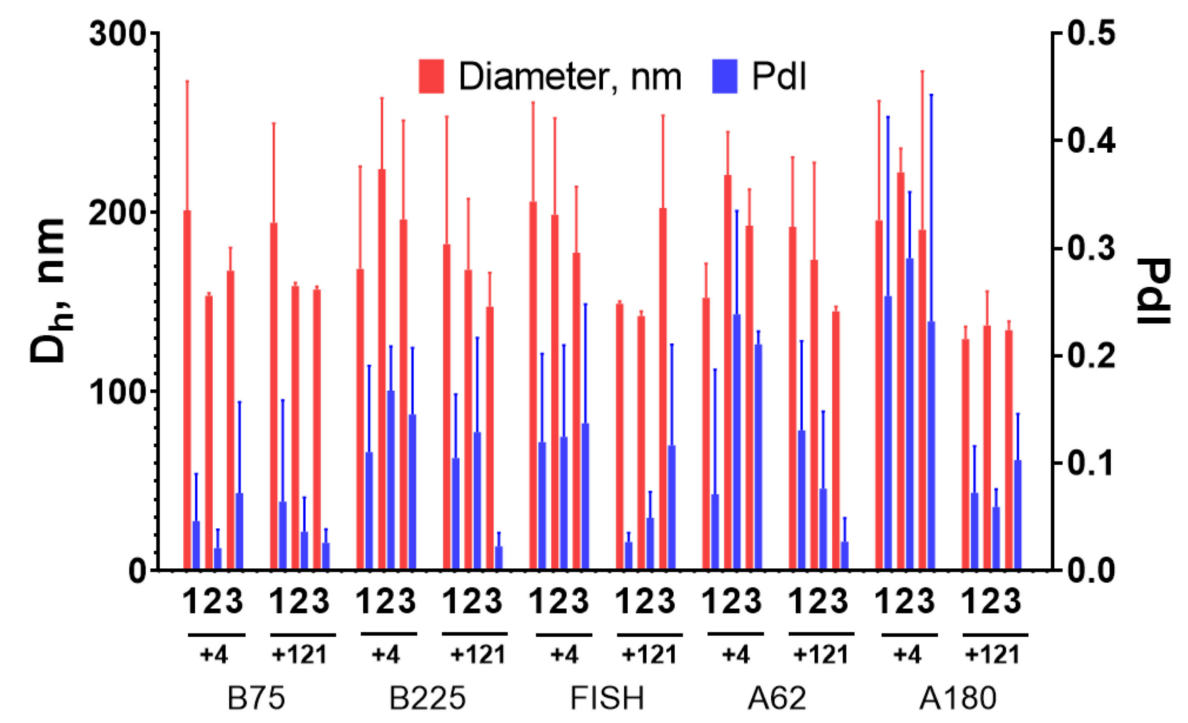

Figure 10. Size (Dh) and polydispersity index (PdI) of non-autoclaved (+4) and autoclaved (+121) gelatin nanoparticles. Mean values of three technical replicates are shown, mean \pm SD.

The influence of autoclave sterilization on the integrity of gelatin nanoparticles was studied by two methods.

Firstly, we measured the concentration of free protein in nanoparticle suspensions before and after autoclaving. Nanoparticles were pelleted by centrifugation, and supernatants were analyzed by bicinchoninic acid assay. Generally, after autoclave sterilization, the percentage of free gelatin molecules did not exceed 1-2\% of total nanoparticle weight, which is, though, 2-5-fold higher in comparison with untreated nanoparticles (Figure 11A). Partial degradation and aggregation of gelatin nanoparticles upon autoclaving are in line with previous reports [68].

Secondly, we assessed the turbidity of nanoparticle preparations. Recently, Geh with colleagues observed a pronounced decrease of turbidity after sterilization of gelatin nanoparticles in the autoclave [68]. For almost all batches of nanoparticles, slight (not more than $8 \%$ ) decrease in turbidity was observed. Both changes in size and dissolution could affect turbidity (Figure 11C). Taking into account DLS data and free protein change, we suppose that dissolution of a small percentage of nanoparticles can take place during sterilization.

Another issue is a chemical alteration caused by sterilization. Autoclaving leads to a decrease of cross-linking degree and an increase of free amino group content in gelatin nanoparticles [68]. Indeed, the zeta potential of sterilized nanoparticles was a bit more positive (by 1-2 mV) compared to plain nanoparticles (Figure 11B). Moreover, the color of nanoparticle suspensions changed after sterilization (Figure S21). The formation of new chemical bonds in gelatin nanoparticles was indirectly assessed by studying their fluorescence behavior at different excitation wavelengths. We compared emission spectra of gelatin nanoparticles before and after sterilization. Excitation wavelengths varied from 260 to $560 \mathrm{~nm}$. Emission spectra recorded at excitation wavelengths of 300, 400, and $500 \mathrm{~nm}$ are presented in Figure S22. Excitation at 300 and $400 \mathrm{~nm}$ resulted in a strong increase of emission, whereas excitation at $500 \mathrm{~nm}$ had almost no effect. These results confirm that some structural alterations accompanied autoclaving. Change of emission was not caused by evaporation of nanoparticle suspension, otherwise, an increase of emission should also 
be observed after excitation at $500 \mathrm{~nm}$. Moreover, the volume of nanoparticle suspension was controlled in the course of the experiment.
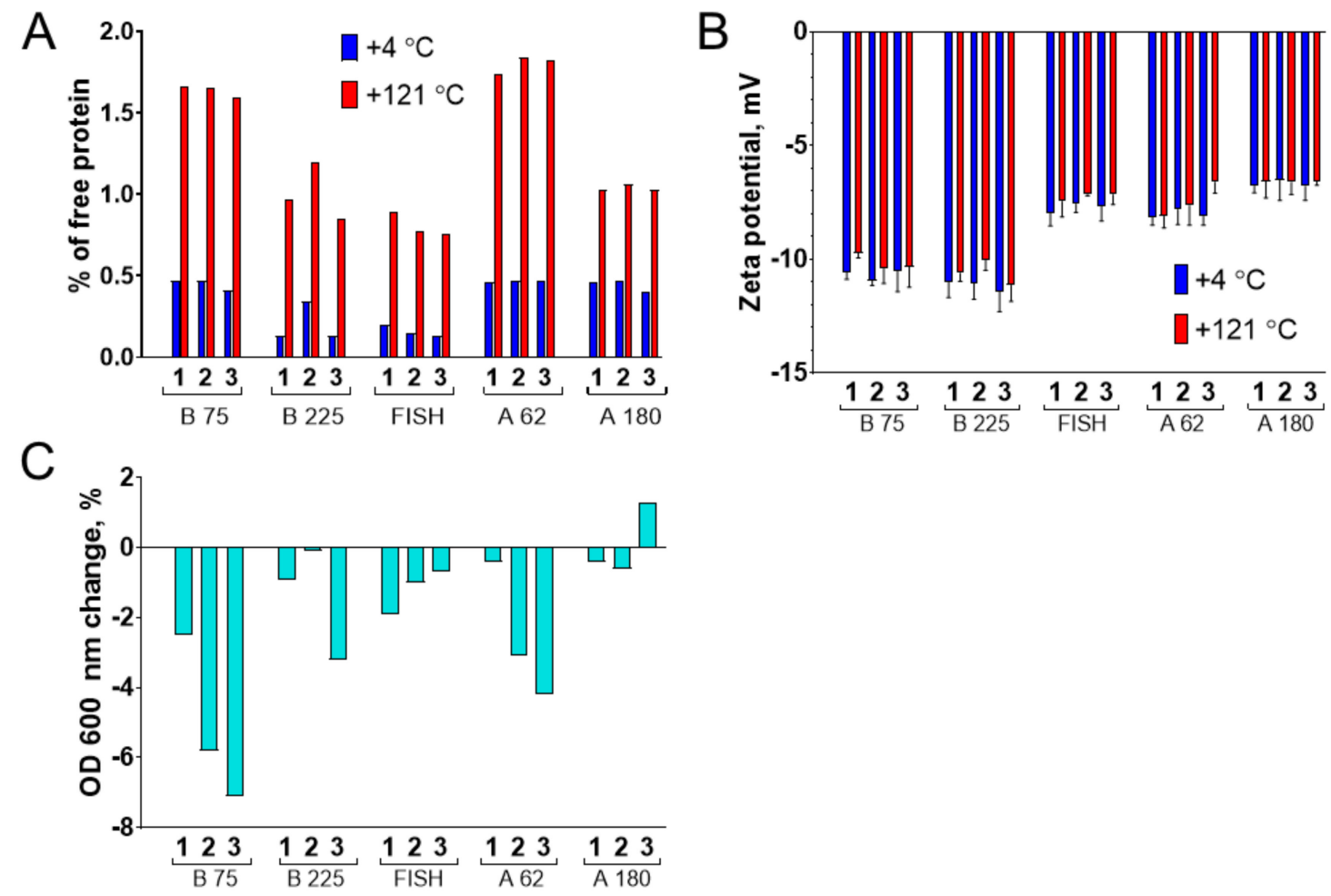

Figure 11. Properties of sterilized $\left(+121^{\circ} \mathrm{C}\right)$ and non-sterilized $\left(+4^{\circ} \mathrm{C}\right)$ nanoparticles. (A) The percentage of free protein in relation to nanoparticle concentration; (B) zeta potential at a $\mathrm{pH} 7$; (C) change of turbidity of the gelatin nanoparticle suspensions after sterilization. Mean zeta potential values of three technical replicates are shown, mean \pm SD.

Although autoclaved samples retained their key properties, we should note that autoclaving of gelatin nanoparticles is appropriate mainly for food applications or in other fields which do not require parenteral usage of nanoparticles. Autoclaving kills microorganisms but does not remove endotoxin, which can interfere with cell and tissue culturing and provokes immune system activation or even endotoxin shock in animals [69]. Autoclaving may be useful for applications where the presence of a small percentage of aggregates and free protein is acceptable. For pharmaceutical purposes, the whole synthesis can be performed in aseptic conditions with endotoxin-free reagents. Another option is post-synthesis depyrogenation and sterilization by gamma-irradiation [72], which was shown to be compatible with gelatin nanoparticle formulations.

\subsection{Effect of Gelatin Nanoparticles on the Viability of Peripheral Blood Mononuclear Cells}

Application of gelatin nanoparticles in drug/gene/antigen delivery or bioimaging requires their blood circulation, making interaction with blood components unavoidable. Therefore, the toxicity of gelatin nanoparticles towards blood immune cells was studied. Peripheral blood mononuclear cells (PBMC) are a complex mixture of cells with a round-shaped nucleus comprising regulatory and effector cells, namely T cells, B cells, NK cells, and monocytes. Being primary cells, they represent the reaction of human blood cells to nanoparticles in a more natural way in comparison with available lymphocyte cell lines [73]. PBMC were isolated from the blood of three healthy donors and incubated with glycine-quenched sterilized gelatin nanoparticles for $24 \mathrm{~h}$. Dead cells were stained with propidium iodide and quantified by flow cytometry. The range of gelatin nanoparticles concentrations was based on literature data, indicating that the concentration of nanoparti- 
cles in the blood can reach values of hundreds of micrograms per milliliter after parenteral administration [74].

The viability of PBMC was higher than $88 \%$ in a whole range of nanoparticle concentrations, indicating low cytotoxicity of gelatin nanoparticles (Figure 12). However, we should note that nanoparticles engulfed or absorbed by cells interfere with fluorescence measurements. Nanoparticles emit fluorescence between 543 and $627 \mathrm{~nm}$ (Figure S23) (propidium iodide detection channel), moreover, at higher nanoparticle concentrations PBMC uptake (or absorb) more nanoparticles as can be seen by the increase of cell granularity (which was assessed by measuring the side scattering of cells) (Figure S9). Therefore, a more thorough study of gelatin nanoparticle toxicity is to be made in the future.

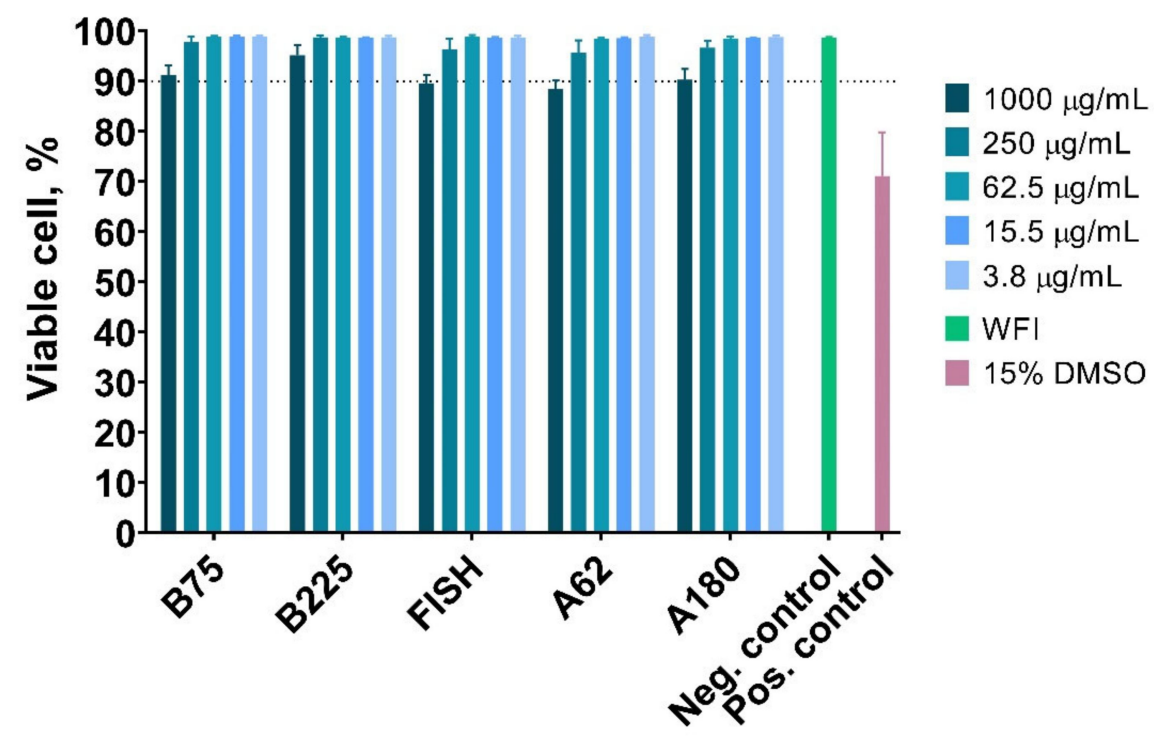

Figure 12. Viability of PBMC in the presence of various concentrations of gelatin nanoparticles. WFI-water for injection. PBMC from three volunteers were obtained, mean $\pm \mathrm{SD}$.

\subsection{Future Perspectives and Limitations of This Study}

The modified desolvation method makes it possible to synthesize nanoparticles from gelatin regardless of its origin and bloom number. This method can be a facile alternative to the commonly utilized two-step desolvation method. Noteworthy, in the course of nanoparticle synthesis, we used common laboratory equipment: centrifuge with a capacity less than $500 \mathrm{~mL}$, thermostat, water bath, and sonicator. All the reagents used are readily available and cheap. Synthesis procedures are simple and can be performed even by non-trained personnel.

One can conclude that the proposed method works well in a laboratory, but is it suitable for large-scale nanoparticle manufacturing? To meet the needs of market consumers, nanoparticles manufacturing rates should be as high as kilograms per day $[75,76]$. Theoretically, this goal can be achieved by a straightforward hundredfold increase of synthesis volumes and by performing multiple parallel syntheses. Undoubtedly, volume increase can lead to a change of nanoparticles size, yield, and their properties caused by mass transfer rate alterations [76]. However, we emphasized that at certain gelatin concentrations mixing rate has a very small effect on nanoparticles size. Moreover, as one can see, gelatin does not aggregate being quickly mixed with a large volume of non-solvent (in contrast to, say, bovine serum albumin). In our opinion, these factors favor the scaling-up of the proposed modified desolvation method.

Recently, direct addition of non-solvent to gelatin under gentle mixing has been realized in the microfluidics-assisted method [77]. This approach allows continuous manufacturing of gelatin nanoparticles. Microfluidic devices enable large-scale synthesis of nanoparticles and better control over their preparation [78,79], however, scaling-up can 
be challenging as it requires chip parallelization or channel diameter increase [39]. The high costs of setting up the microfluidic devices for industrial scale-up, as well as technical issues (e.g., channels clogging $[78,80]$ ), are among potential barriers to their implementation. Thus, despite numerous advantages of microfluidics technology, batch methods (including modified desolvation method) are still attractive in terms of their industrial application [39].

In this study, glutaraldehyde was utilized for nanoparticle cross-linking. Despite being effective and widely available, it raises concerns about its potential toxicity (both as a component of nanoparticles and a component of waste). Therefore, the toxicity of gelatin nanoparticles stabilized with glutaraldehyde needs to be thoroughly tested. Moreover, other approaches to gelatin cross-linking should be considered, e.g., reagentless irradiative cross-linking [81] or stabilization by polymer entrapment [3].

The environmental impact of all nanoparticle synthesis components should also be considered before their translation into practice [82]. The main concerns are usually related to the toxicity of cross-linking agents, however, proper management of organic waste comprising organic solvent itself and products of its interaction with the cross-linking agent can also be a problem. More cheap and effective (from the synthesis point of view) solvent can be less appropriate in terms of disposal, therefore, different combinations of desolvating agents (not necessarily organic solvents!) and cross-linking agents are to be tested. Here, we used short-chain alcohols as desolvating agents. They can be classified as environmentally favorable solvents [83]. However, other organic solvents or other approaches to desolvation (e.g., salting out), which can be potentially more effective or safe, need to be studied in the future. Moreover, testing of different cross-linking agents or non-solvents is of importance, because some substances to be loaded in gelatin nanoparticles can be incompatible with specific synthesis conditions, i.e., non-soluble in particular desolvating agent, unstable at high $\mathrm{pH}$, and so on.

In conclusion, we need to mention several limitations of the present study:

1. The size of all synthesized gelatin nanoparticles exceeded $100 \mathrm{~nm}$. We did not obtain smaller nanoparticles, however, the desolvation method allows the preparation of nanoparticles whose diameter is less than $100 \mathrm{~nm}[23,26]$. We suppose that a decrease of initial gelatin concentration or increase of gelatin solution $\mathrm{pH}$ is a possible way to obtain nanoparticles smaller than $100 \mathrm{~nm}$.

2. We did not prepare nanoparticles from gelatin solutions with concentrations higher than $20 \mathrm{mg} / \mathrm{mL}$ at a high scale. As we mentioned in Section 3.3, our goal was to prepare relatively small nanoparticles, less than $200 \mathrm{~nm}$, which is possible by using smaller gelatin concentrations for all tested gelatin types. Data obtained in the course of optimization experiments and our previous results [30] both demonstrate that gelatin nanoparticles can be prepared at high starting gelatin concentrations. Variation of $\mathrm{pH}$ and volume of the desolvating agent is a possible way to decrease the size of nanoparticles when the concentration of gelatin is high.

3. As we mentioned before, we did not remove endotoxin from gelatin nanoparticles nor examine endotoxin concentration in nanoparticle preparations prior to cell viability testing. Synthesis of apyrogenic nanoparticles is a challenging task, which requires a separate set of experiments and was, therefore, beyond the scope of the present work. We just note that the protocol of gelatin depyrogenation was previously reported by Singh et al. [37], besides, post-synthesis depyrogenation by gamma-irradiation also remains a possible option [72].

4. The effect of several factors on the desolvation process was not studied: temperature of starting materials [49], salt concentration, acidic $\mathrm{pH}$ values, gelatin pre-incubation [84], the longevity of incubation with alcohols, and so on. Nevertheless, we performed preliminary experiments adding $\mathrm{NaCl}$ before desolvation. The addition of salt resulted in the formation of microparticles visible by the eye, however, a systematic study has not been conducted. 
5. Despite various types of animal gelatin being tested, we did not prepare nanoparticles from human recombinant gelatin. Application of natural gelatin from animal sources can be limited due to pathogen (first of all, prions) contamination, religious reasons, and its potential immunogenicity [4]. In previous papers, gelatin nanoparticles synthesized from recombinant human gelatin by one-step desolvation method were described [23], therefore, we believe that the universal nature of the proposed method enables usage of human recombinant gelatin as a starting material.

\section{Conclusions}

In this work, a simple and scalable modified desolvation method to fabricate gelatin nanoparticles was proposed. The method allows the preparation of gelatin nanoparticles with sizes 100-300 $\mathrm{nm}$ with a high yield on the hundreds-of-milligram scale. The resulting nanoparticles possess excellent colloidal stability, low cytotoxicity, and can be loaded with hydrophobic molecules, being appropriate for application in food science, drug and vaccine delivery, cell culture, and immunoassays development.

Supplementary Materials: The following are available online at https:/ /www.mdpi.com/article/10 .3390 / pharmaceutics13101537/s1, Optimization experiments (methods and results); Figure S1: Size of gelatin nanoparticles desolvated with ethanol; Figure S2: Yield of gelatin nanoparticles desolvated with ethanol; Figure S3: Size of gelatin nanoparticles prepared at different conditions using isopropyl alcohol as a poor solvent; Figure S4: Yield of gelatin nanoparticles prepared at different conditions using isopropyl alcohol as a poor solvent; Figure S5: Size of gelatin nanoparticles prepared at different conditions using ethanol as a poor solvent; Figure S6: Yield of gelatin nanoparticles prepared at different conditions using ethanol as a poor solvent; Figure S7: Fluorescence of monocytes engulfing particles; Figure S8: Gating of living and dead cells; Figure S9: SSC histograms of monocyte gate; Figure S10: Glass vials and magnets after synthesis of gelatin nanoparticles; Figure S11: Gelatin nanoparticles prepared under stirring from the mixtures of gelatins A; Figure S12: Gelatin nanoparticles prepared from gelatin A, 62 bloom by one-time addition of isopropyl alcohol; Figure S13: Size distribution of B75-1 nanoparticles obtained by TEM; Figure S14: Absorbance and fluorescence of B75-1 nanoparticles; Figure S15: Emission spectra of gelatin nanoparticles at various excitation wavelengths; Figure S16: Zeta potential of gelatin nanoparticles; Figure S17: Colloidal stability of gelatin nanoparticles at different salt concentrations; Figure S18: Storage stability of gelatin nanoparticles; Figure S19: Emission and excitation spectra of gelatin nanoparticles loaded with europium complexes; Figure S20: Size distribution of europium loaded gelatin nanoparticles; Figure S21: Color of autoclaved and non-autoclaved gelatin nanoparticle suspensions; Figure S22: Emission spectra of non-autoclaved and autoclaved gelatin nanoparticles; Figure S23: Fluorescence intensity of gelatin nanoparticles in the passband of detection filter. Table S1: Literature data on yields of desolvation and nanoprecipitation methods. References [85-99] are cited in the Supplementary Materials.

Author Contributions: Formal analysis, P.K. and O.B.; investigation, P.K., O.B., S.L., A.N., M.B., V.T., D.K. and A.M.; visualization, P.K.; writing—original draft, P.K.; resources, S.Z. and M.R.; conceptualization, S.Z. and M.R.; writing-review and editing. S.Z., P.K. and M.R.; funding acquisition, P.K. All authors have read and agreed to the published version of the manuscript.

Funding: The reported study was funded by RFBR and Kaliningrad Oblast according to the research project № 19-415-393005 (preparation of fluorescence gelatin nanoparticles and study of their fluorescent properties), by RFBR research project 19-015-00408 (preparation of gelatin nanoparticles by the desolvation method), and by Ministry of Science and Higher Education of the Russian Federation within the framework of the Russian State Assignment under contract No. AAAA-A19-1191122900107 (assessment of nanoparticles cytotoxicity).

Institutional Review Board Statement: Research was approved by the Review Board of the Institute of Ecology and Genetics of Microorganisms UB RAS (IRB00010009).

Informed Consent Statement: All procedures were performed in accordance with the 1964 Declaration of Helsinki and its later amendments or comparable ethical standards. Written informed consent was obtained from all volunteers. 
Data Availability Statement: The datasets used and/or analyzed during the current study are available from the corresponding author on reasonable request.

Conflicts of Interest: The authors declare that they have no conflict of interest.

\section{References}

1. Gomez-Guillen, M.C.; Gimenez, B.; Lopez-Caballero, M.E.; Montero, M.P. Functional and bioactive properties of collagen and gelatin from alternative sources: A review. Food Hydrocoll. 2011, 25, 1813-1827. [CrossRef]

2. Derkach, S.R.; Voron'ko, N.G.; Kuchina, Y.A.; Kolotova, D.S. Modified Fish Gelatin as an Alternative to Mammalian Gelatin in Modern Food Technologies. Polymers 2020, 12, 3051. [CrossRef] [PubMed]

3. Khan, S.A. Mini-Review: Opportunities and challenges in the techniques used for preparation of gelatin nanoparticles. Pak. J. Pharm. Sci. 2020, 33, 221-228. [PubMed]

4. Alipal, J.; Pu'Ad, N.M.; Lee, T.C.; Nayan, N.; Sahari, N.; Basri, H.; Idris, M.; Abdullah, H. A review of gelatin: Properties, sources, process, applications, and commercialisation. Mater. Today Proc. 2021, 42, 240-250. [CrossRef]

5. Miernicki, M.; Hofmann, T.; Eisenberger, I.; von der Kammer, F.; Praetorius, A. Legal and practical challenges in classifying nanomaterials according to regulatory definitions. Nat. Nanotechnol. 2019, 14, 208-216. [CrossRef]

6. Kianfar, E. Protein nanoparticles in drug delivery: Animal protein, plant proteins and protein cages, albumin nanoparticles. J. Nanobiotechnol. 2021, 19, 159. [CrossRef]

7. Hong, S.; Choi, D.W.; Kim, H.N.; Park, C.G.; Lee, W.; Park, H.H. Protein-Based Nanoparticles as Drug Delivery Systems Pharmaceutics 2020, 12, 604. [CrossRef]

8. Yasmin, R.; Shah, M.; Khan, S.A.; Ali, R. Gelatin nanoparticles: A potential candidate for medical applications. Nanotechnol. Rev. 2017, 6, 191-207. [CrossRef]

9. $\quad$ Esteban-Pérez, S.; Andrés-Guerrero, V.; López-Cano, J.J.; Molina-Martínez, I.; Herrero-Vanrell, R.; Bravo-Osuna, I. Gelatin Nanoparticles-HPMC Hybrid System for Effective Ocular Topical Administration of Antihypertensive Agents. Pharmaceutics 2020, 12, 306. [CrossRef]

10. Sudheesh, M.S.; Vyas, S.P.; Kohli, D.V. Nanoparticle-based immunopotentiation via tetanus toxoid-loaded gelatin and aminated gelatin nanoparticles. Drug Deliv. 2011, 18, 320-330. [CrossRef]

11. Narayanan, D.; Geena, M.G.; Lakshmi, H.; Koyakutty, M.; Nair, S.; Menon, D. Poly-(ethylene glycol) modified gelatin nanoparticles for sustained delivery of the anti-inflammatory drug Ibuprofen-Sodium: An in vitro and in vivo analysis. Nanomedicine 2013, 9, 818-828. [CrossRef]

12. Klier, J.; Bartl, C.; Geuder, S.; Geh, K.J.; Reese, S.; Goehring, L.S.; Winter, G.; Gehlen, H. Immunomodulatory asthma therapy in the equine animal model: A dose-response study and evaluation of a long-term effect. Immun. Inflamm. Dis. 2019, 7, 130-149. [CrossRef] [PubMed]

13. Lin, A.; Liu, Y.; Zhu, X.; Chen, X.; Liu, J.; Zhou, Y.; Qin, X.; Liu, J. Bacteria-Responsive Biomimetic Selenium Nanosystem for Multidrug-Resistant Bacterial Infection Detection and Inhibition. ACS Nano 2019, 13, 13965-13984. [CrossRef] [PubMed]

14. Bello, A.B.; Kim, D.; Kim, D.; Park, H.; Lee, S.-H. Engineering and functionalization of gelatin biomaterials: From cell culture to medical applications. Tissue Eng. Part B 2020, 26, 164-180. [CrossRef] [PubMed]

15. Clark, C.C.; Aleman, J.; Mutkus, L.; Skardal, A. A mechanically robust thixotropic collagen and hyaluronic acid bioink supplemented with gelatin nanoparticles. Bioprinting 2019, 16, e00058. [CrossRef]

16. Diba, M.; Koons, G.L.; Bedell, M.L.; Mikos, A.G. 3D printed colloidal biomaterials based on photo-reactive gelatin nanoparticles. Biomaterials 2021, 274, 120871. [CrossRef] [PubMed]

17. Wei, X.; Chen, K.; Wang, Z.; Huang, B.; Wang, Y.; Yu, M.; Liu, W.; Guo, S.-S.; Zhao, X.-Z. Multifunctional Gelatin Nanoparticle Integrated Microchip for Enhanced Capture, Release, and Analysis of Circulating Tumor Cells. Part. Part. Syst. Charact. 2019, 36 , 1900076. [CrossRef]

18. Feng, X.; Dai, H.; Ma, L.; Fu, Y.; Yu, Y.; Zhou, H.; Guo, T.; Zhu, H.; Wang, H.; Zhang, Y. Properties of Pickering emulsion stabilized by food-grade gelatin nanoparticles: Influence of the nanoparticles concentration. Colloids Surf. B 2020, 196, 111294. [CrossRef]

19. Coester, C.J.; Langer, K.; van Briesen, H.; Kreuter, J. Gelatin nanoparticles by two step desolvation-a new preparation method, surface modifications and cell uptake. J. Microencapsul. 2000, 17, 187-193. [CrossRef]

20. Zwiorek, K. Gelatin Nanoparticles as Delivery System for Nucleotide-Based Drugs. Ph.D. Thesis, Ludwig Maximilian University of Munich, Munich, Germany, 3 August 2006.

21. Ahlers, M.; Coester, C.; Zwiorek, K.; Zillies, J. Nanoparticles and Method for the Production. Thereof. Patent WO2006021367A1, 18 August 2005.

22. Geh, K.J.; Hubert, M.; Winter, G. Optimisation of one-step desolvation and scale-up of gelatine nanoparticle production. J. Microencapsul. 2016, 33, 595-604. [CrossRef]

23. Won, Y.-W.; Kim, Y.-H. Recombinant human gelatin nanoparticles as a protein drug carrier. J. Control. Release 2008, 127, 154-161. [CrossRef] [PubMed]

24. Madkhali, O. Cationic Gelatin/Pluronic-based Nanoparticles as Novel Non-Viral Delivery Systems for Gene Therapy. Ph.D. Thesis, University of Waterloo, Waterloo, ON, Canada, 2018.

25. Stevenson, A.T.; Lewis, S.A.; Whittington, A.R. Filtration initiated selective homogeneity (FISH) desolvation: A new method to prepare gelatin nanoparticles with high physicochemical consistency. Food Hydrocoll. 2018, 84, 337-342. [CrossRef] 
26. Shamarekh, K.S.; Gad, H.A.; Soliman, M.E.; Sammour, O.A. Towards the production of monodisperse gelatin nanoparticles by modified one step desolvation technique. J. Pharm. Invest. 2020, 50, 189-200. [CrossRef]

27. Kaul, G.; Amiji, M. Long-circulating poly(ethylene glycol)-modified gelatin nanoparticles for intracellular delivery. Pharm. Res. 2002, 19, 1061-1067. [CrossRef]

28. Kommareddy, S.; Amiji, M.M. Preparation and Loading of Gelatin Nanoparticles. Cold Spring Harb. Protoc. 2008, 2, pdb-prot4885. [CrossRef]

29. Ofokansi, K.; Winter, G.; Fricker, G.; Coester, C. Matrix-loaded biodegradable gelatin nanoparticles as new approach to improve drug loading and delivery. Eur. J. Pharm. Biopharm. 2010, 76, 1-9. [CrossRef]

30. Khramtsov, P.; Kalashnikova, T.; Bochkova, M.; Kropaneva, M.; Timganova, V.; Zamorina, S.; Rayev, M. Measuring the concentration of protein nanoparticles synthesized by desolvation method: Comparison of Bradford assay, BCA assay, hydrolysis/UV spectroscopy and gravimetric analysis. Int. J. Pharm. 2021, 15, 120422. [CrossRef]

31. Beyer, C.; Claisen, L. Ueber die Einführung von Säureradicalen in Ketone. Ber. Dtsch. Chem. Ges. 1887, 20, 2178. [CrossRef]

32. Mironov, L.Y.; Parfenov, P.S.; Shurukhina, A.V.; Lebedev, Y.I.; Metlenko, A.A. Delayed Fluorescence of Dyes Sensitized by Eu ${ }^{3+}$ Chelate Nanoparticles. J. Phys. Chem. C 2017, 121, 19958-19965. [CrossRef]

33. De Abreu Costa, L.; Henrique Fernandes Ottoni, M.; Dos Santos, M.G.; Meireles, A.B.; Gomes de Almeida, V.; de Fátima Pereira, W.; Alves de Avelar-Freitas, B.; Eustáquio Alvim Brito-Melo, G. Dimethyl Sulfoxide (DMSO) Decreases Cell Proliferation and TNF- $\alpha$, IFN- $\gamma$, and IL-2 Cytokines Production in Cultures of Peripheral Blood Lymphocytes. Molecules 2017, 22, 1789. [CrossRef]

34. Shin, H.; Kwak, M.; Lee, T.G.; Lee, J.Y. Quantifying the level of nanoparticle uptake in mammalian cells using flow cytometry. Nanoscale 2020, 12, 15743-15751. [CrossRef]

35. Farrugia, C.A.; Groves, M.J. Gelatin Behaviour in Dilute Aqueous Solution: Designing a Nanoparticulate Formulation. J. Pharm. Pharmacol. 1999, 51, 643-649. [CrossRef]

36. Vandervoort, J.; Ludwig, A. Preparation and evaluation of drug-loaded gelatin nanoparticles for topical ophthalmic use. Eur. J. Pharm. Biopharm. 2004, 57, 251-261. [CrossRef]

37. Singh, A.; Xu, J.; Mattheolabakis, G.; Amiji, M. EGFR-targeted gelatin nanoparticles for systemic administration of gemcitabine in an orthotopic pancreatic cancer model. Nanomed. Nanotechnol. Biol. Med. 2016, 12, 589-600. [CrossRef]

38. Von Storp, B.; Engel, A.; Boeker, A.; Ploeger, M.; Langer, K. Albumin nanoparticles with predictable size by desolvation procedure. J. Microencapsul. 2012, 29, 138-146. [CrossRef]

39. Matthew, S.A.L.; Totten, J.D.; Phuagkhaopong, S.; Egan, G.; Witte, K.; Perrie, Y.; Seib, F.P. Silk Nanoparticle Manufacture in Semi-Batch Format. ACS Biomater. Sci. Eng. 2020, 14, 6748-6759. [CrossRef]

40. Mehravar, R.; Jahanshahi, R.; Najafpour, G.D.; Saghatoleslami, N. Applying the Taguchi method for optimized fabrication of $\alpha$-lactalbumin nanoparticles as carrier in drug delivery and food science. Iran. J. Energy Environ. 2011, 2, 87-91.

41. Subara, D.; Jaswir, I.; Alkhatib, M.F.R.; Noorbatcha, I.A. Synthesis of fish gelatin nanoparticles and their application for the drug delivery based on response surface methodology. Adv. Nat. Sci. Nanosci. Nanotechnol. 2018, 9, 045014. [CrossRef]

42. Abdelrady, H.; Hathout, R.M.; Osman, R.; Saleem, I.; Mortada, N.D. Exploiting gelatin nanocarriers in the pulmonary delivery of methotrexate for lung cancer therapy. Eur. J. Pharm. Sci. 2019, 133, 115-126. [CrossRef]

43. Pei, Y.; Zheng, Y.; Li, Z.; Liu, J.; Zheng, X.; Tang, K.; Kaplan, D.L. Ethanol-induced coacervation in aqueous gelatin solution for constructing nanospheres and networks: Morphology, dynamics and thermal sensitivity. J. Colloid Interface Sci. 2021, 582, 610-618. [CrossRef] [PubMed]

44. El-Sayed, N.; Trouillet, V.; Clasen, A.; Jung, G.; Hollemeyer, K.; Schneider, M. NIR-Emitting Gold Nanoclusters-Modified Gelatin Nanoparticles as a Bioimaging Agent in Tissue. Adv. Healthc. Mater. 2019, 8, 1900993. [CrossRef] [PubMed]

45. Subara, D.; Jaswir, I. The Effect of Processing Parameters on the Properties of Fish Gelatin Hydrolysate Nanoparticle. J. Sci. Appl. Technol. 2021, 1, 17-24. [CrossRef]

46. Seib, F.P.; Jones, G.T.; Rnjak-Kovacina, J.; Lin, Y.; Kaplan, D.L. pH-dependent anticancer drug release from silk nanoparticles. Adv. Healthc. Mater. 2013, 2, 1606-1611. [CrossRef] [PubMed]

47. Morel, M.-H.; Redl, A.; Guilbert, S. Mechanism of Heat and Shear Mediated Aggregation of Wheat Gluten Protein upon Mixing. Biomacromolecules 2002, 3, 488-497. [CrossRef]

48. Balthasar, S. Charakterisierung Proteinbasierter Nanopartikel Zum Transport von Oligonukleotiden für eine Rezeptor-Vermittelte Zellaufnahme. Ph.D. Thesis, Goethe-Universität Frankfurt am Main, Frankfurt, Germany, 2005. Available online: http://d-nb. info/978388593/34 (accessed on 15 July 2021).

49. Azarmi, S.; Huang, Y.; Chen, H.; McQuarrie, S.; Abrams, D.; Roa, W.; Finlay, W.H.; Miller, G.G.; Löbenberg, R. Optimization of a two-step desolvation method for preparing gelatin nanoparticles and cell uptake studies in $143 \mathrm{~B}$ osteosarcoma cancer cells. $J$. Pharm. Pharm. Sci. 2006, 9, 124-132.

50. Arroyo-Maya, I.J.; Rodiles-López, J.O.; Cornejo-Mazón, M.; Gutiérrez-López, G.F.; Hernández-Arana, A.; Toledo-Núñez, C.; Barbosa-Cánovas, G.V.; Flores-Flores, J.O.; Hernández-Sánchez, H. Effect of different treatments on the ability of $\alpha$-lactalbumin to form nanoparticles. J. Dairy Sci. 2012, 95, 6204-6214. [CrossRef]

51. Sun, S.; Xiao, Q.-R.; Wang, Y.; Jiang, Y. Roles of alcohol desolvating agents on the size control of bovine serum albumin nanoparticles in drug delivery system. J. Drug Deliv. Sci. Technol. 2018, 47, 193-199. [CrossRef]

52. Mohammad-Beigi, H.; Shojaosadati, S.A.; Morshedi, D.; Mirzazadeh, N.; Arpanaei, A. The Effects of Organic Solvents on the Physicochemical Properties of Human Serum Albumin Nanoparticles. Iran. J. Biotechnol. 2016, 14, 45-50. [CrossRef] 
53. Nixon, J.R.; Khalil, S.A.H.; Carless, J.E. Phase relationships in the simple coacervating system isoelectric gelatin: Ethanol: Water. J. Pharm. Pharmacol. 1966, 18, 409-416. [CrossRef]

54. Ahsan, S.M.; Rao, C.M. The role of surface charge in the desolvation process of gelatin: Implications in nanoparticle synthesis and modulation of drug release. Int. J. Nanomed. 2017, 12, 795-808. [CrossRef]

55. Ding, M.; Zhang, T.; Zhang, H.; Tao, N.; Wang, X.; Zhong, J. Effect of preparation factors and storage temperature on fish oil-loaded crosslinked gelatin nanoparticle pickering emulsions in liquid forms. Food Hydrocoll. 2019, 95, 326-335. [CrossRef]

56. Spoljaric, S.; Ju, Y.; Caruso, F. Protocols for Reproducible, Increased-Scale Synthesis of Engineered Particles-Bridging the "Upscaling Gap". Chem. Mater. 2021, 33, 1099-1115. [CrossRef]

57. Langer, K.; Anhorn, M.G.; Steinhauser, I.; Dreis, S.; Celebi, D.; Schrickel, N.; Faust, S.; Vogel, V. Human serum albumin (HSA) nanoparticles: Reproducibility of preparation process and kinetics of enzymatic degradation. Int. J. Pharm. 2008, 347, 109-117. [CrossRef]

58. Lowry, G.V.; Hill, R.J.; Harper, S.; Rawle, A.F.; Hendren, C.O.; Klaessig, F.; Nobbmann, U.; Sayre, P.; Rumble, J. Guidance to improve the scientific value of zeta-potential measurements in nanoEHS. Environ. Sci. Nano 2016, 3, 953-965. [CrossRef]

59. Balthasar, S.; Michaelis, K.; Dinauer, N.; Von Briesen, H.; Kreuter, J.; Langer, K. Preparation and characterisation of antibody modified gelatin nanoparticles as drug carrier system for uptake in lymphocytes. Biomaterials 2005, 26, 2723-2732. [CrossRef]

60. Leo, E.; Vandelli, M.A.; Cameroni, R.; Forni, F. Doxorubicin-loaded gelatin nanoparticles stabilized by glutaraldehyde: Involvement of the drug in the cross-linking process. Int. J. Pharm. 1997, 155, 75-82. [CrossRef]

61. Cai, B.; Rao, L.; Ji, X.; Bu, L.L.; He, Z.; Wan, D.; Yang, Y.; Liu, W.; Guo, S.; Zhao, X.Z. Autofluorescent gelatin nanoparticles as imaging probes to monitor matrix metalloproteinase metabolism of cancer cells. J. Biomed. Mater. Res. Part A 2016, 104, 2854-2860. [CrossRef]

62. Wei, W.; Wang, L.-Y.; Yuan, L.; Wei, Q.; Yang, X.-D.; Su, Z.-G.; Ma, G.-H. Preparation and Application of Novel Microspheres Possessing Autofluorescent Properties. Adv. Funct. Mater. 2007, 17, 3153-3158. [CrossRef]

63. Tsai, Y.-J.; Hu, C.-C.; Chu, C.-C.; Imae, T. Intrinsically fluorescent PAMAM dendrimer as gene carrier and nanoprobe for nucleic acids delivery: Bioimaging and transfection study. Biomacromolecules 2011, 12, 4283-4290. [CrossRef]

64. Singh, S.K.; Singh, M.K.; Nayak, M.K.; Kumari, S.; Grácio, J.J.A.; Dash, D. Size distribution analysis and physical/fluorescence characterization of graphene oxide sheets by flow cytometry. Carbon 2011, 49, 684-692. [CrossRef]

65. Sivera, M.; Kvitek, L.; Soukupova, J.; Panacek, A.; Prucek, R.; Vecerova, R.; Zboril, R. Silver nanoparticles modified by gelatin with extraordinary $\mathrm{pH}$ stability and long-term antibacterial activity. PLoS ONE 2014, 9, e103675. [CrossRef]

66. Fuchs, S. Gelatin Nanoparticles as a Modern Platform for Drug Delivery: Formulation Development and Immunotherapeutic Strategies. Ph.D. Thesis, Ludwig Maximilian University of Munich, Munich, Germany, 29 July 2010.

67. Shilpi, D.; Kushwah, V.; Agrawal, A.K.; Jain, S. Improved Stability and Enhanced Oral Bioavailability of Atorvastatin Loaded Stearic Acid Modified Gelatin Nanoparticles. Pharm. Res. 2017, 34, 1505-1516. [CrossRef]

68. Geh, K. Gelatine Nanoparticles as Immunomodulatory Drug Delivery System: Advanced Production Processes and Clinical Trials. Ph.D. Thesis, Ludwig Maximilian University of Munich, Munich, Germany, 16 March 2018. [CrossRef]

69. Vetten, M.A.; Yah, C.S.; Singh, T.; Gulumian, M. Challenges facing sterilization and depyrogenation of nanoparticles: Effects on structural stability and biomedical applications. Nanomed. Nanotechnol. Biol. Med. 2014, 10, 1391-1399. [CrossRef]

70. Ma, X.; Hargrove, D.; Dong, Q.; Song, D.; Chen, J.; Wang, S.; Lu, X.; Cho, Y.K.; Fan, T.-H.; Lei, Y. Novel green and red autofluorescent protein nanoparticles for cell imaging and in vivo biodegradation imaging and modeling. RSC Adv. 2016, 6, 50091-50099. [CrossRef]

71. Langevin, D.; Raspaud, E.; Mariot, S.; Knyazev, A.; Stocco, A.; Salonen, A.; Luch, A.; Haase, A.; Trouiller, B.; Relier, C.; et al. Towards reproducible measurement of nanoparticle size using dynamic light scattering: Important controls and considerations. NanoImpact 2018, 10, 161-167. [CrossRef]

72. Monti, F.; Manfredi, G.; Palamà, I.E.; Kovtun, A.; Zangoli, M.; D’Amone, S.; Ortolani, L.; Bondelli, G.; Szreder, T.; Bobrowski, K.; et al. Sterilization of Semiconductive Nanomaterials: The Case of Water-Suspended Poly-3-Hexylthiophene Nanoparticles. Adv. Healthc. Mater. 2021, 10, 2001306. [CrossRef] [PubMed]

73. Jeong, H.; Hwang, J.; Lee, H.; Hammond, P.T.; Choi, J.; Hong, J. In vitro blood cell viability profiling of polymers used in molecular assembly. Sci. Rep. 2017, 7, 9481. [CrossRef]

74. Ong, Y.R.; De, R.; Johnston, A.P.R. In Vivo Quantification of Nanoparticle Association with Immune Cell Subsets in Blood. Adv. Healthc. Mater. 2021, 10, 2002160. [CrossRef]

75. A matter of scale. Nat. Nanotechnol. 2016, 11, 733. [CrossRef]

76. Feng, J.; Markwalter, C.E.; Tian, C.; Armstrong, M.; Prud'homme, R.K. Translational formulation of nanoparticle therapeutics from laboratory discovery to clinical scale. J. Transl. Med. 2019, 17, 200. [CrossRef]

77. Van Den Broek, S.; Nieuwland, P.J.; Kaspar Koch, K. Continuous Flow Production of Gelatin Nanoparticles. U.S. Patent US9289499B2, 22 March 2016.

78. Chiesa, E.; Dorati, R.; Pisani, S.; Conti, B.; Bergamini, G.; Modena, T.; Genta, I. The Microfluidic Technique and the Manufacturing of Polysaccharide Nanoparticles. Pharmaceutics 2018, 10, 267. [CrossRef]

79. Zhang, L.; Chen, Q.; Ma, Y.; Sun, J. Microfluidic Methods for Fabrication and Engineering of Nanoparticle Drug Delivery Systems. ACS Appl. Bio. Mater. 2020, 3, 107-120. [CrossRef] 
80. Niculescu, A.-G.; Chircov, C.; Bîrcă, A.C.; Grumezescu, A.M. Nanomaterials Synthesis through Microfluidic Methods: An Updated Overview. Nanomaterials 2021, 11, 864. [CrossRef]

81. Varca, G.H.C.; Queiroz, R.G.; Lugão, A.B. Irradiation as an alternative route for protein crosslinking: Cosolvent free BSA nanoparticles. Radiat. Phys. Chem. 2016, 124, 111-115. [CrossRef]

82. Egorova, K.S.; Galushko, A.S.; Ananikov, V.P. Introducing tox-Profiles of Chemical Reactions. Angew. Chem. Int. Ed. Engl. 2020, 59, 22296-22305. [CrossRef]

83. Capello, C.; Fischer, U.; Hungerbühler, K. What is a green solvent? A comprehensive framework for the environmental assessment of solvents. Green Chem. 2007, 9, 927. [CrossRef]

84. Vinjamuri, B.P.; Papachrisanthou, K.; Haware, R.V.; Chougule, M.B. Gelatin solution $\mathrm{pH}$ and incubation time influences the size of the nanoparticles engineered by desolvation. J. Drug Deliv. Sci. Technol. 2021, 63, 102423. [CrossRef] 